\title{
Differential Phanerozoic evolution of cratonic and non-cratonic lithosphere from a thermochronological perspective: São Francisco Craton and marginal orogens (Brazil)
}

\author{
Ana Carolina Liberal Fonseca ${ }^{\mathrm{a}, *}$, Tiago Amâncio Novo ${ }^{\mathrm{a}}$, Simon Nachtergaele ${ }^{\mathrm{b}}$, Tobias M.R. Fonte-Boa ${ }^{\mathrm{a}}$, \\ Gerben Van Ranst ${ }^{\mathrm{b}}$, Johan De Grave ${ }^{\mathrm{b}}$ \\ a Universidade Federal de Minas Gerais, Programa de Pós-Graduação em Geologia, CPMTC-IGC, Campus Pampulha, Belo Horizonte, MG, Brazil \\ ${ }^{\mathrm{b}}$ Department of Geology, Ghent University, Ghent, Belgium
}

\section{A R T I C L E I N F O}

\section{Article history:}

Received 10 March 2020

Received in revised form 20 December 2020

Accepted 19 January 2021

Available online 24 January 2021

Handling Editor: T. Gerya

\section{Keywords:}

Brasília Orogen

Araçuaí Orogen

Continental lithosphere rigidity

Lithospheric inheritance

Apatite fission track thermochronology

\begin{abstract}
A B S T R A C T
The São Francisco Craton (SFC) and its marginal Araçuaí and Brasília orogens exhibit a significant diversity in their lithospheric architecture. These orogens were shaped during the Neoproterozoic-Cambrian amalgamation of West Gondwana. The rigid cratonic lithosphere of the SFC and the relatively weak lithosphere of the Araçuaí Orogen were disrupted during the Cretaceous opening of the South Atlantic Ocean, whereas the Brasilia Orogen remained in the continental hinterland. In earlier research, the thermal effects of the Phanerozoic reactivations in the shallow crust of the Araçuaí Orogen have been revealed by low-temperature thermochronology, mainly by apatite fission track (AFT) analysis. However, analyses from the continental interior are scarce. Here we present new AFT data from forty-three samples from the Brasilia Orogen, the SFC and the Araçuaí Orogen, far from the passive margin of the Atlantic coast ( 150 to $800 \mathrm{~km})$. Three main periods of basement exhumation were identified: (i) Paleozoic, recorded both by samples from the SFC and Brasília Orogen; (ii) Early Cretaceous to Cenomanian, recorded by samples from the Araçuaí Orogen; and (iii) Late Cretaceous to Paleocene, inferred in samples from all domains. We compare the differential exhumation pattern of the different geotectonic provinces with their lithospheric strengths. We suggest that the SFC likely concentrated the Meso-Cenozoic reactivations in narrow weak zones while the Araçuaí Orogen displayed a far-reaching Meso-Cenozoic deformation. The Brasília Orogen seems to be an example of a stronger orogenic lithosphere, inhibiting reworking, confirmed by our new AFT data. Understanding the role of the lithosphere rigidity may be decisive to comprehend the processes of differential denudation and the tectonic-morphological evolution over Phanerozoic events.
\end{abstract}

(c) 2021 International Association for Gondwana Research. Published by Elsevier B.V. All rights reserved.

\section{Introduction}

During the evolution of the continental lithosphere, lithospheric strength plays a significant role in controlling the initiation and character of extensional and compressional deformation (Kusznir and Park, 1984). Rifting, tectonic reactivation and reworking are mainly concentrated in segments of weak lithospheres and pre-existing shear zones (Manatschal et al., 2015; Petersen and Schiffer, 2016; Schiffer et al., 2019; Tommasi and Vauchez, 1997). In contrast, rigid and stiff lithosphere, such as characteristic of cratons, is more resilient to be affected by major tectonic events (Artemieva, 2006). Several main factors contribute strongly to lithosphere rigidity: (i) mantle and crust rheology; (ii) geothermal gradient; (iii) crustal thickness; (iv) lower crustal

\footnotetext{
* Corresponding author.

E-mail addresses: anacarolina.LiberalFonseca@ugent.be (A.C.L. Fonseca), Simon.Nachtergaele@ugent.be (S. Nachtergaele),gerben.VanRanst@ugent.be (G. Van Ranst), Johan.DeGrave@ugent.be (J. De Grave).
}

composition; and (v) tectonic inheritance (Kusznir and Park, 1984; Manatschal et al., 2015; Salazar-Mora et al., 2018). Orogenic lithosphere is usually considered favorable for reworking due to its fertile content, high concentration in radiogenic elements, and its mantle anisotropy array. However, Krabbendam (2001) described how some orogens can produce strong lithosphere and inhibit deformation. The West Gondwana break-up during the Early Cretaceous seems to have followed the structural lineaments of ancient mountain belts (e.g. Araçuaí Orogen) (Tommasi and Vauchez, 2001; Autin et al., 2013; Reuber and Mann, 2019; Will and Frimmel, 2018), although the rifting also broke cratonic areas (e.g. the Bahia cratonic bridge of the São FranciscoCongo Craton) whereas it preserved other orogenic belts (e.g. Brasília Orogen).

Low-temperature thermochronology has been used to constrain the reactivations in different tectonic environments (e.g. Fernie et al., 2018; Gillespie et al., 2017; Mackintosh et al., 2019), due to its ability to identify cooling/heating episodes that affected the shallow crust. Episodes of high cooling rates are usually connected to moments of crustal 
deformation (i.e. uplift and flexural bending followed by denudation) that are determined by reaction of the lithosphere to a stress source. In Brazil, several thermochronological studies have been conducted along the Atlantic passive margins (e.g. Cogné et al., 2011; Engelmann de Oliveira et al., 2016; Gallagher et al., 1995; Hackspacher et al., 2004; Hueck et al., 2019; Jelinek et al., 2014; Japsen et al., 2012; Krob et al., 2020; Van Ranst et al., 2020a; Harman et al., 1998), mainly focused on detecting thermal variations during rift and post-rift events that are pointed out as essential in the formation of the coastal relief and landscape along the Brazilian Atlantic coast. The cooling phases identified by the previous works indicate that uplift must have taken place during Meso-Cenozoic times, inducing the erosion and exhumation of the crystalline basement. However, the hinterland response to the Cretaceous rifting is still poorly understood in South America (Heine et al., 2013; Pérez-Díaz and Eagles, 2014; Zalán, 2004).

In south-eastern Brazil, the São Francisco Craton (SFC) and its marginal belts are part of the South American Platform, (Almeida et al., 1981; Almeida et al., 2000; Brito Neves, 2002). The cratonic area preserves the Archean nucleus and segments of a Paleoproterozoic orogen, both partially covered by Proterozoic sedimentary successions and few Phanerozoic deposits (Heilbron et al., 2017). During the Neoproterozoic until the early Paleozoic, various continental collision-accretion events shaped the preceding paleocontinent throughout the Brasiliano orogenic cycle that eventually resulted in the amalgamation of West Gondwana. The portion of the Precambrian basement not involved in deformation triggered by the Brasiliano cycle defines the SFC (Almeida et al., 1981; Alkmim et al., 1993; Alkmim, 2015). To the west, the Brasília Orogen (Fig. 1) was assembled in the course of the closure of a wide ocean during the subduction of continental units, tectonic accretion events and associated nappe exhumation (Valeriano, 2017). It was stabilized relatively early (600-560 Ma) when compared with the other orogenic fronts that surrounded the SFC and afterwards it remained confined in the hinterland of continental blocks, i.e. West Gondwana and South America continents (Pimentel, 2016). To the east of the SFC, the Araçuaí-West Congo (AWCO) Orogen (Fig. 1) was formed following the closure of a fairly narrow ocean branch. This was then succeeded by continental collision, and orogenic collapse during the onset of the Neoproterozoic up to the Cambrian-Ordovician boundary (Pedrosa-Soares et al., 2008). The AWCO was disrupted and split into two counterparts with the incipient opening of the South Atlantic Ocean. It currently composes a segment of the Brazilian and Congo passive margins.

As the SFC and its marginal belts are composed of a representative diversity of lithosphere architectures, understanding of their thermal evolution after the Phanerozoic stabilization can clarify the role of the lithospheric strength in their exhumation history. Moreover, the Phanerozoic upper crust thermal evolution of the SFC hinterland domains and the Brasília Orogen still remains poorly constrained. In this study we present the results of apatite fission track (AFT) thermochronometry applied to forty-three samples collected in three transects crossing the structural fabric of the Araçuaí Orogen, SFC and Brasília Orogen. We aim to constrain the exhumation phases of each terrain, investigating the possible relationships of cooling/heating episodes to geodynamic processes and their surface expressions. From these, we can evaluate if there is a differential exhumation pattern between the terrains and if it is related to the lithospheric characteristics of each tectonic province.

\section{Geological setting}

The study area comprises the SFC and two of its marginal orogenic belts, i.e. the Araçuaí and Brasília orogens, which surrounded and shaped the craton during the Ediacaran - Cambrian amalgamation of West Gondwana. Segments of SFC and Araçuaí Orogen constitute a portion of the Brazilian passive margin and were connected to their African counterparts, Congo Craton and AWCO fold-and-thrust belt, respectively, before Pangea (including West Gondwana) break-up in the
Early Cretaceous (Fig. 1) (Hasui et al., 1975; Almeida, 1977; Trompette et al., 1992; Alkmim and Teixeira, 2017). On the western side of the SFC, the Brasília Orogen has been part of continental interior since the Cambrian (Fig. 1).

\section{1. (Pre)Cambrian basement}

The SFC basement was formed originally by an Archean nucleus, that is represented by TTG-type granitoids as the result of granitegreenstone terrains amalgamation (Heilbron et al., 2017; Alkmim, 2004). The Archean nucleus was attached, on its eastern side (present-day co-ordinates), to Paleoproterozoic orogenic segments: The Eastern Bahia Orogenic Domain, the Mineiro Belt, and the so-called Quadrilátero Ferrífero (Iron Quadrangle) mineral province (Fig. 1) (Heilbron et al., 2017; Alkmim and Teixeira, 2017; Barbosa and Barbosa, 2017). The Paleoproterozoic orogenic system and the Archean nucleus are remnants of the São Francisco-Congo paleo-continent. This paleo-continent was affected by anorogenic magmatism and taphrogenic processes which created intracratonic basins and passivemargin sedimentation at the end of the Paleoproterozoic (MartinsNeto et al., 2001). During the Ediacaran - Cambrian, several continental collisions affected the São Francisco-Congo Craton and led to the final configuration of West Gondwana with the inversion of the previous extensional rifts. This process resulted in the Brasiliano - Pan-African orogenic systems that are used to delineate the boundaries of the craton. Due to its long and complex tectonic history, as well as the large area covered by Phanerozoic sediments, the SFC's structural framework is still not completely understood. In SE Brazil, the craton is surrounded by three Brasiliano orogenic fronts, the Araçuaí, Ribeira and Brasília orogens (Fig. 1) (Almeida, 1977).

The Araçuaí Belt is located to the southeast of the SFC (Fig. 1) where it underlies the Brazilian Atlantic margin. To the south of the Araçuaí Belt, around Lat $21^{\circ} \mathrm{S}$, it merges with the Ribeira Belt (Fig. 1). The Araçuaí Belt encompasses an entire magmatic arc, an ophiolite sliver, syn- and post-collisional magmatic bodies, and a suture zone (Pedrosa-Soares et al., 2008). The orogen was formed due to the closure of the Adamastor ocean during the amalgamation of West Gondwana (Heilbron et al., 2008). The regional structural trend diverts from NNE to NE from north to south (Fig. 1). The metamorphic grade of exposed units ranges from greenschist to amphibolite facies, with metamorphic peak ages of 600-580 Ma and final collision at 540 to $530 \mathrm{Ma}$ (Alkmim et al., 2017). The orogenic collapse induced post-collisional magmatism and a variety of extensional structures and is dated to have occurred at around 530-490 Ma (Pedrosa-Soares et al., 2001, 2008; Marshak et al., 2006). The Araçuaí Orogen has been coined a hot orogen (e.g. Cavalcante et al., 2018; Fossen et al., 2017; Vauchez et al., 2007, 2019; Richter et al., 2016) as it is characterized by high temperatures, slow cooling and the emplacement of large volumes of magma during its accretionary, syn- and post-collisional phases (Fossen et al., 2017; Vauchez et al., 2007, 2019).

The Brasília Orogen was developed along the western margin of the SFC (Almeida et al., 1981) during the convergence of the Amazonian, São Francisco-Congo and Paranapanema paleocontinents (Pimentel et al., 2004), that consumed a large oceanic slab (Brito Neves, 1999). The fold belt comprises Archean - Paleoproterozoic rocks, which, together with a Proterozoic metasedimentary cover, a juvenile magmatic arc (Goiás arc), and granitic complexes, were folded and thrusted toward the SFC (Pimentel et al., 2000). In its southern portion, a NW-SE structural trend prevails, while in the southernmost area, the regional trend is overlapped by W-vergent nappes and transitional structures of the Ribeira Belt (Valeriano, 2017a). Peak metamorphism is constrained at 650-630 Ma with amphibolite to granulite facies conditions (e.g. Moraes et al., 2002; Piuzana, 2003; Reno et al., 2009; Valeriano et al., 2004). Analysis from medium-temperature thermochronometers $\left({ }^{40} \mathrm{Ar} /{ }^{39} \mathrm{Ar}\right.$ and $\mathrm{K} / \mathrm{Ar}$ in biotite and mica) indicates an exhumation and cooling of the Brasília Orogen between 600 and 


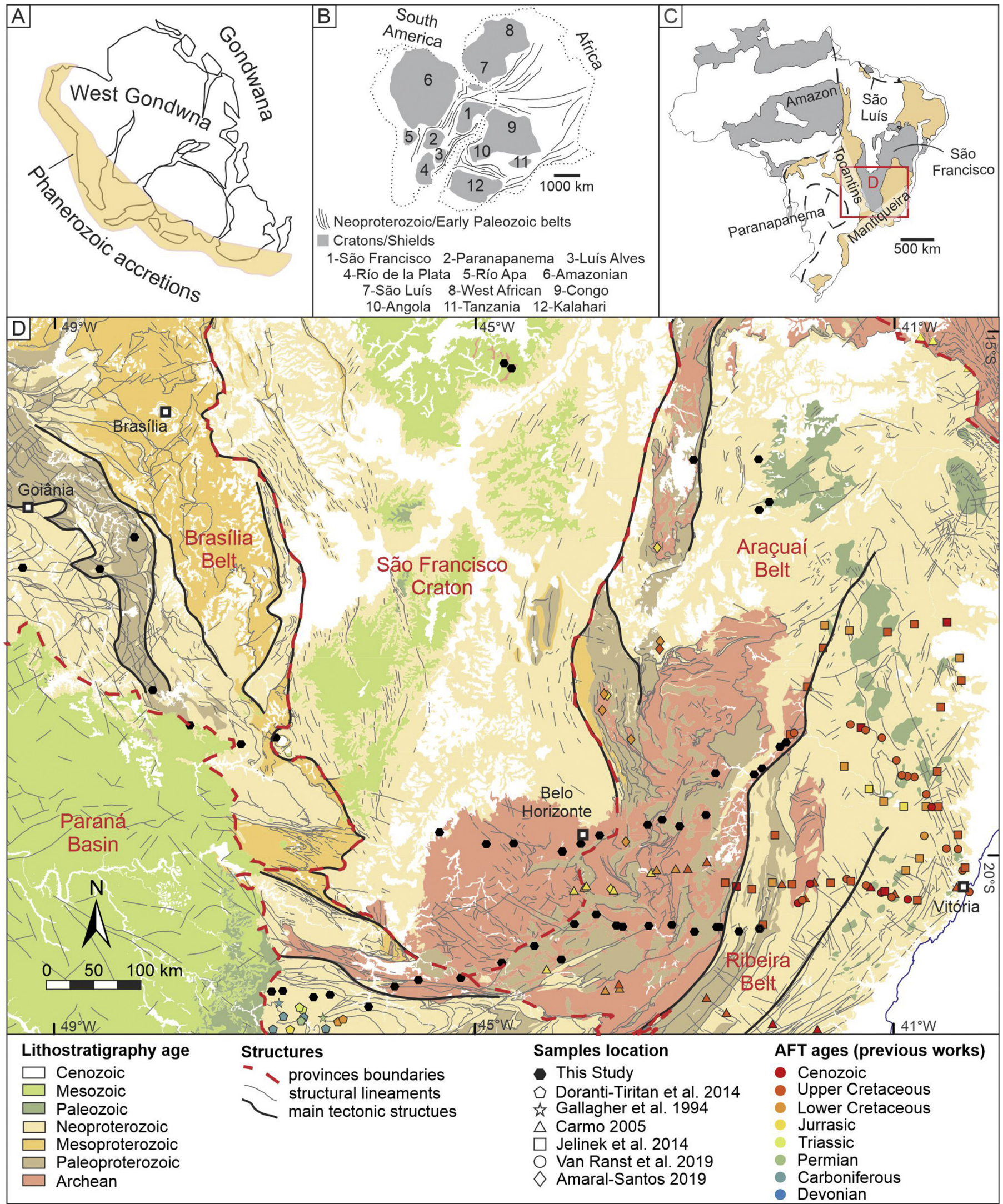

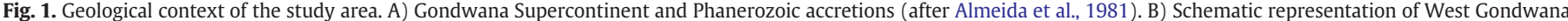

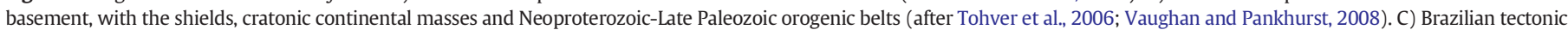

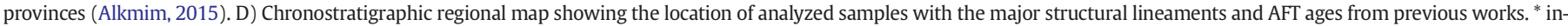
dicates samples from Fonseca et al. (2020). 
$560 \mathrm{Ma}$ as its orogenic collapse progressed (Hasui and Almeida, 1970; Valeriano et al., 2000; Pimentel et al., 2004).

\subsection{Post-Cambrian Evolution and existing thermochronological data}

Following the cessation of the Brasiliano orogenic cycle, the West Gondwana continental interior went through a consolidation stage, forming a relatively stable platform during the early Paleozoic (Carneiro et al., 2012; Zalán, 2004). Scarce low-temperature thermochronological data on the SFC and its margins, have revealed a pronounced Paleozoic cooling period in the southern Brasília Orogen (Doranti-Tiritan et al., 2014; Gallagher et al., 1994; Ribeiro et al., 2005) and in the northeastern SFC (Jelinek et al., 2014; Japsen et al., 2012). These authors tentatively relate the cooling event with far-field effects of the long-lived orogeny at the southern margin of Gondwana, i.e. the
Famatian and Gondwanic orogenic cycles (Fig. 1, Fig. 2: 1 to 4, Jelinek et al., 2014), the deposition of sedimentary sequences in the adjoining Paraná Basin (Fig. 2: 10 to 12, Ribeiro et al., 2005) and widespread Carboniferous glaciation (Doranti-Tiritan et al., 2014).

During the Paleozoic, the Famatian (Early Ordovician to Early Carboniferous) and Gondwanic (Early Carboniferous to middle Permian) orogenic cycles took place in the SW portion of the West Gondwana paleo-continent (Fig. 1-A) (Heredia et al., 2018, and references therein) by the docking of continental fragments (collisional type) and by subduction of oceanic terranes (non-collisional type). From their analyses of tectonic subsidence curves of the Paraná-Chaco Basin and its sedimentary record, Milani and Ramos (1998) proposed that the extensional tectonic subsidence episodes that affected this basin were closely related to the onset of these Paleozoic geodynamic cycles. The Paleozoic deposits in the Paraná-Chaco Basin preserve marine, fluvial,
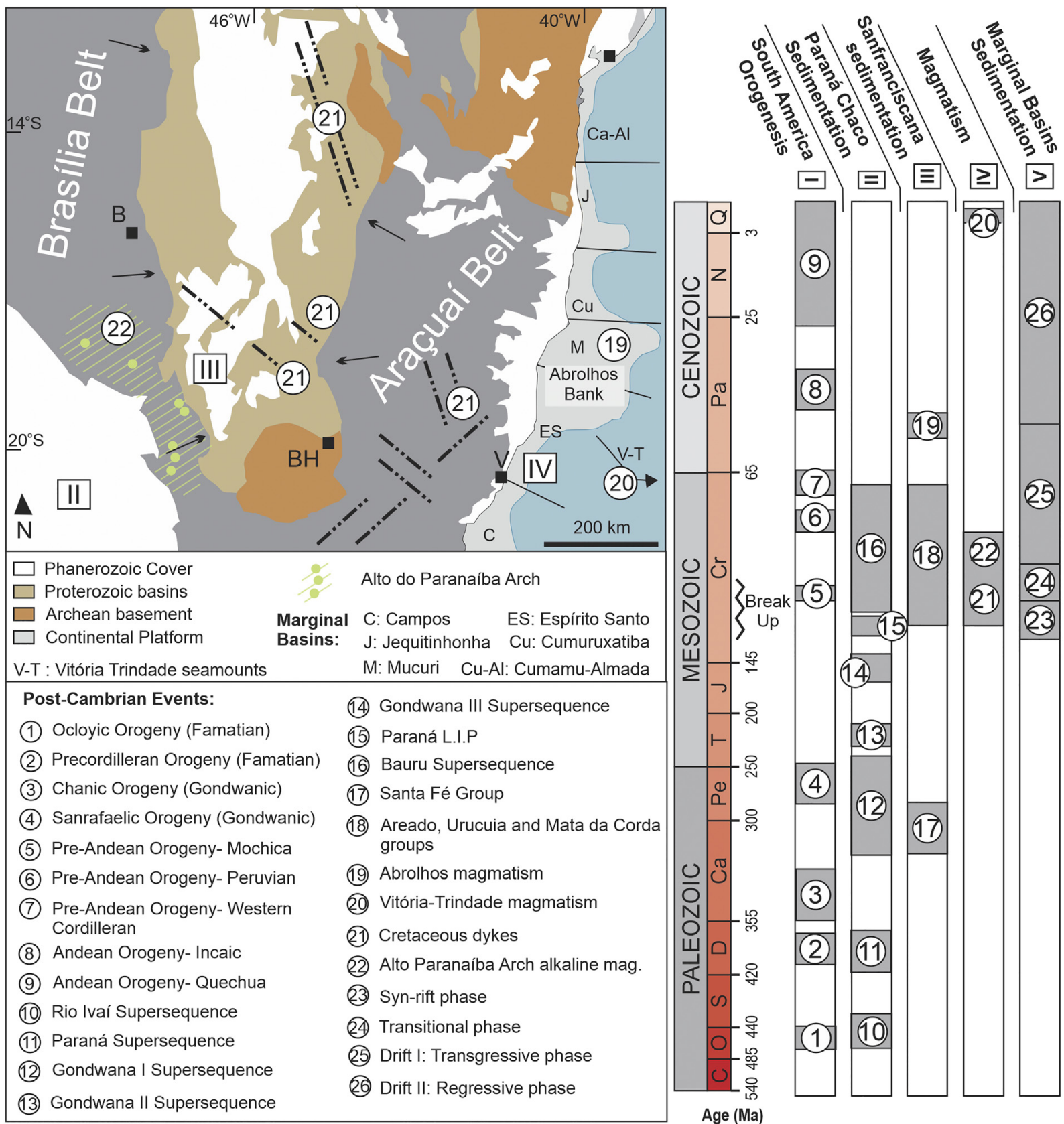

Fig. 2. Main post-Cambrian tectonic, sedimentary and volcanic events (after Zalán, 2004). 
and coastal sediments, covering a large area $\left(1,500,000 \mathrm{~km}^{2}\right)$ with thicknesses up to $4500 \mathrm{~m}$ (Fig. 2: 10 to 12, Milani et al., 2007). The Carboniferous glaciation event is recorded by the Itararé Group in this basin and by striated surfaces in its basement (Fig. 2: 12, Buso et al., 2017; Caputo and Crowell, 1985; Eyles et al., 1993; Griffis et al., 2018; Montañez and Poulsen, 2013; Mottin et al., 2018; Visser, 1987). The sedimentary record in the Sanfranciscana Basin of the SFC, initiates with Carboniferous glacial deposits (Santa Fé Group, Fig. 2: 17) (Sgarbi et al., 2001; Limarino et al., 2014; Torsvik and Cocks, 2013; Vesely and Assine, 2006; Reis et al., 2017). The Carboniferous glacial period was followed by increasingly arid conditions as reflected in sediments from in the Paleozoic - Mesozoic transition.

During the Mesozoic, the relative stability of the West Gondwana hinterland was disturbed by taphrogenic processes culminating in the complete continental break-up and eventual opening of the South Atlantic Ocean. Preceding the rifting processes, some magmatic events occurred that are mainly well-expressed in the African counterpart. According to Pérez-Díaz and Eagles (2014), separation started at the latest by $138 \mathrm{Ma}$ in the far south and then it propagated northwards describing a counterclockwise rotation. The onset of the break-up was approximately coeval with the emplacement of the Paraná-Etendeka flood basalts in the Paraná Basin (Fig. 2: 15, Hueck et al., 2018; Krob et al., 2020; Rocha et al., 2020). Krob et al. (2020) combined stratigraphic records and thermochronological data sets to suggest a possible thermal effect of this mantle plume in the intraplate environment adjacent to its emplacement. Also, during this time, the Transminas mafic dike swarm (Coelho and Chaves, 2017) intruded the basement of the SFC and the western Araçuaí Orogen (Fig. 2: 21, Chaves, 2013; Rosset et al., 2007). Besides this activity, tectonic reactivation and mafic intrusions took place in the Cambrian Colatina Fracture Zone, in the eastern Araçuaí Orogen (Fig. 2: 21, Santiago et al., 2019; Mendes, 2017). A reheating effect of these magmatic events in the hosting basement has hitherto not been observed (Amaral-Santos et al., 2019; Van Ranst et al., 2020a).

Structural inheritance possibly guided the South Atlantic rifting, since it tended to follow the Brasiliano - Pan African structures (e.g. de Wit et al., 2008; Misra and Mukherjee, 2015; Nemčok, 2016; Tommasi and Vauchez, 2001; Vauchez et al., 1997; Will and Frimmel, 2018). The rheology of the rift-resistant cratonic nucleus led to the development of deep faults and narrow sedimentary basins, in contrast to wide and evaporite-rich basins evolving on the Brasiliano belts (Fig. 2, see platform size differences in the map, e.g. Alkmim, 2004; Mohriak et al., 2008). The marginal basins record the tectonic phases of the rifting evolution as syn-rift, transitional and drift phases (Fig. 221 to 24, e.g. Asmus, 1982). The rifting reached and affected the São Francisco-Congo Craton and AWCO basement at ca. 120 to $112 \mathrm{Ma}$ (Torsvik et al., 2009) effectively separating the SFC and Araçuaí Orogen from its African counterparts (Congo craton and West Congo Belt). The uplift and consequential erosion of the rift shoulders are pointed out as the reasons of Early Cretaceous basement cooling inferred by AFT analyses in the Araçuaí Orogen (Jelinek et al., 2014; Van Ranst et al., 2020a).

The post-rift evolution is marked by significant volcanic activity, mainly between the Late Cretaceous and the Paleogene. In the continental hinterland, series of alkaline intrusions were emplaced in the Alto Paranaíba Arch in the Brasília Orogen (Fig. 2: 20, e.g. Riccomini et al., 2005). Several authors suggest a new pulse of sedimentation in the Paraná (Bauru Supersequence, Fig. 2: 16, Batezelli, 2017; Milani et al., 2007) and Sanfranciscana basins (Areado Fm., Fig. 2: 18, Campos and Dardenne, 1997; Uhlein et al., 2011) linked to pulses of doming and denudation connected to these intrusions. Offshore Brazil, the Abrolhos Bank, which forms a remarkable off-shore platform (Fig. 2: 19), records a sedimentary-volcanic complex developed during this time (Mohriak and Fainstein, 2012).

Late Cretaceous to Paleogene rapid cooling of the regional basement is widely recorded by low-temperature thermochronometry (AFT and $\mathrm{U}-\mathrm{Th} / \mathrm{He}$ ) in the Araçuaí Orogen (Carmo, 2005; Jelinek et al., 2014;
Van Ranst et al., 2020a). The authors interpreted the cooling as the effect of erosion of an uplifted area. This hypothesis is supported by the significant increment of the marginal basin sedimentary thicknesses during this time. Multiple uplift driving forces seem to have acted (e.g. isostasy, tectonic basement reactivations and mantle drag), however the distinct role of each one is still unclear. Van Ranst et al. (2020a) suggest the influence of a thermal anomaly possibly related to the hypothetical Trindade mantle plume and the offshore magmatism (Thompson et al., 1998) which may have weakened the lower crust, hence making it more susceptible to tectonic basement reactivations due to far-field stresses. The stresses may have been triggered by a complex interaction of forces that compress the South American Plate between the Andean collision zone and the ridge-push of the Atlantic Ocean spreading zone (e.g. Japsen et al., 2012; Cogné et al., 2012). The authors highlighted the synchronism between this Araçuaí Orogen denudation phase and the Peruvian stage of the Andean orogeny (Figs. 2-6) and thus they proposed this collision stage as the main source of lithospheric stress. Jelinek et al. (2014) also emphasize the role of crustal weakening by mantle heating but pointed at isostasy as main uplift process.

\section{Samples and method}

In order to investigate the Phanerozoic thermochronological evolution of the SFC and its surrounded mobile belts, this study applied the AFT method (Appendix A) on the Precambrian-Cambrian basement. Forty-three samples of granites and gneisses were collected following three approximately E-W oriented transects: S(outh), C(entral) and $\mathrm{N}$ (orth) (Fig. 1-D). These transects were selected to cross the structural fabric established during the Brasiliano orogeny cycle, and cover the three tectonic provinces where only few data, if any, were previously acquired (Fig. 1-D). A lower sampling resolution of basement outcrops in the SFC is due to the extensive Phanerozoic sediment cover which reduces the amount of possible sampling sites. Twenty-four samples are from the Araçuaí Orogen, twelve samples from the SFC and seven samples are from the Brasília Orogen. Details of the lithology, elevation and geographical locations of each sample are provided in Table 1. Apatite grains were concentrated by conventional crushing and magnetic and heavy liquid separation in Centro de Pesquisas professor Manoel Teixeira da Costa (CPMTC), Belo Horizonte (Brazil).

For this study we applied the AFT external detector method (Hurford and Green, 1982; Fleisher et al., 1975) based on the standard procedures used at the fission track laboratory at Ghent University (described by e.g. De Pelsmaeker et al., 2015; Nachtergaele et al., 2020; Van Ranst et al., 2020a). Grains (150-250 per sample) were hand-picked and mounted in Struers CaldoFix-2 epoxy resin, after which they were grinded on SiC paper and polished using diamond suspension $(6 \mu \mathrm{m}$, $3 \mu \mathrm{m}$ and $1 \mu \mathrm{m}$ ). Apatite mounts were etched in $5.5 \mathrm{~mol} / \mathrm{L} \mathrm{HNO}_{3}$ solution during $20 \mathrm{~s}$ at $21^{\circ} \mathrm{C}$ (Donelick, 1991) in order to make the spontaneous fission tracks detectable through optical microscopy. Thermal neutron irradiation was carried out at the Belgian Nuclear Research Centre (SCK, Mol) using the Belgian Reactor 1 (BR1) facility (Channel X26; De Grave et al., 2010). Age standards (Durango and Fish Canyon Tuff) were included in the irradiation package for zeta-calibration (Hurford and Green, 1983). Thermal neutron fluence was monitored by four IRMM-540 dosimeter glasses (De Corte et al., 1998), which were regularly spread over the package. Induced fission tracks were revealed in the irradiated external detectors (ED) (Goodfellow low-U muscovite) using $40 \% \mathrm{HF}$ for $40 \mathrm{~min}$ at $21{ }^{\circ} \mathrm{C}$.

Age calculations were performed using the overall weighted mean zeta (OWMZ). The majority of the samples were analyzed by one analyst $(\zeta=313.9 \pm 5.8, \mathrm{AF}$ ) using the Leica DM 2500P microscope equipped with a Leica DFC 295 camera. Using a different microscope and optical set-up, samples S1, S3, S11 and S13 were analyzed by TN $(\zeta=254.6 \pm 8.8)$ and samples $5,10, \mathrm{C} 3, \mathrm{~S} 4$ and $\mathrm{S} 14$ by SN $(\zeta=$ $286.2 \pm 4.7$ ), both under the motorized Nikon Eclipse Ni-E microscope with a Nikon DS-Ri2 camera attachment (Van Ranst et al., 2020b). To 


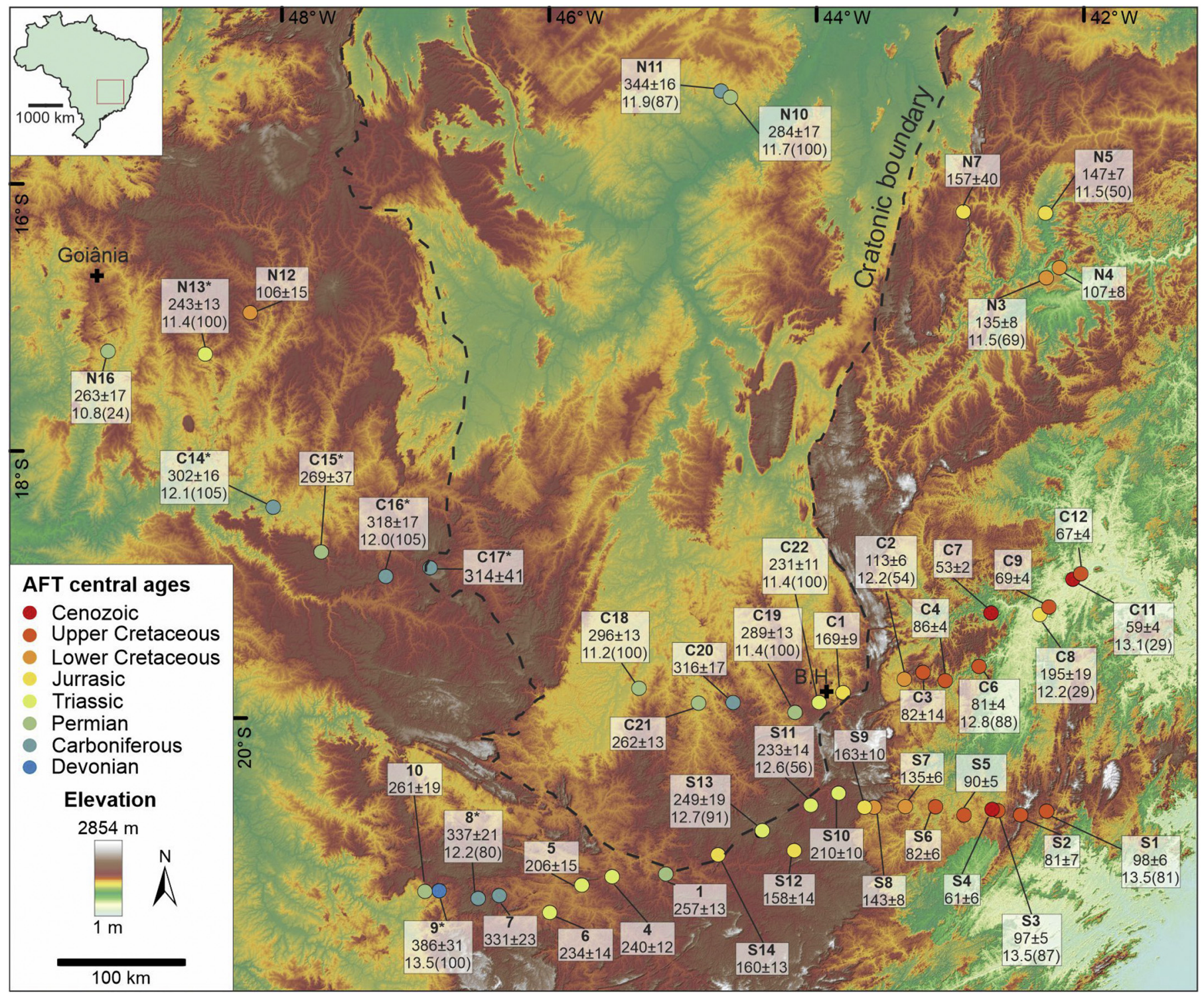

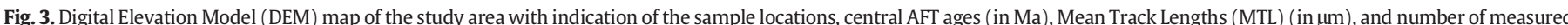
confined track lengths in brackets. For general location see Fig. 1. * indicates samples from Fonseca et al. (2020). BH = Belo Horizonte.

assess the track density, we aimed to count tracks in at least 20 grains for each sample. Central ages and Chi-squared $\left(\chi^{2}\right)$ homogeneity test parameters (Galbraith, 2005; Galbraith, 1981; Galbraith and Laslett, 1993) were calculated using "IsoplotR" software (Vermeesch, 2018) and are reported in Table 2.

The length-frequency distributions were acquired by ideally measuring 100 (sub)horizontal confined tracks in each sample. All track lengths and the angles between the track and the crystallographic caxis were measured with the Nikon Eclipse Ni-E microscope with a DS-Ri2 camera attached at a $1000 \times$ magnification. The etch pit diameter parallel to the crystallographic c-axis $\left(D_{\text {par }}\right)$ (Donelick et al., 1999) was measured (100-200 per sample) using a the $D_{\text {par }}$ module within Nikon NIS-Elements AR (Van Ranst et al., 2020b). Data from samples with a sufficient number of track lengths $(>50)$ were used as input in the QTQt software (Gallagher, 2012) to perform the Markov Chain Monte Carlo (MCMC) inverse modeling with $10^{5}$ pre- and post-burn in iterations. We opted not to use c-axis projection because the anisotropy of our samples is anomalously low, causing c-axis projection to 'overcorrect' and invert anisotropy. (Van Ranst et al., 2020a). Only the present-day temperature was constrained as $(25 \pm 15){ }^{\circ} \mathrm{C}$ in order to not bias the models. The prior for the MCMC run was set to the (central age \pm central age) for time and, $(70 \pm 70){ }^{\circ} \mathrm{C}$ for temperature.

\section{Results}

We present a new AFT dataset, which is summarized in Table 2, of 43 analyzed samples from the Araçuaí Orogen, the SFC, and the Brasília Orogen. AFT central ages and confined track lengths from twelve analyzed samples are geographically displayed in Fig. 3. Swath elevation profiles (north A-B; central C-D; south E-F) with added samples position and AFT central ages are presented in Fig. 4. Supplementary figures on analytical results (among others comparative diagrams showing the differential data of the different terrains studied) are to be found in Appendix B. Samples C3 and S4 failed the Chi-squared test $\left(P\left(\chi^{2}\right)<5 \%\right)$. Samples C8, C11 and N16 yielded insufficient confined tracks $(n<50)$ to reconstruct thermal history models. 

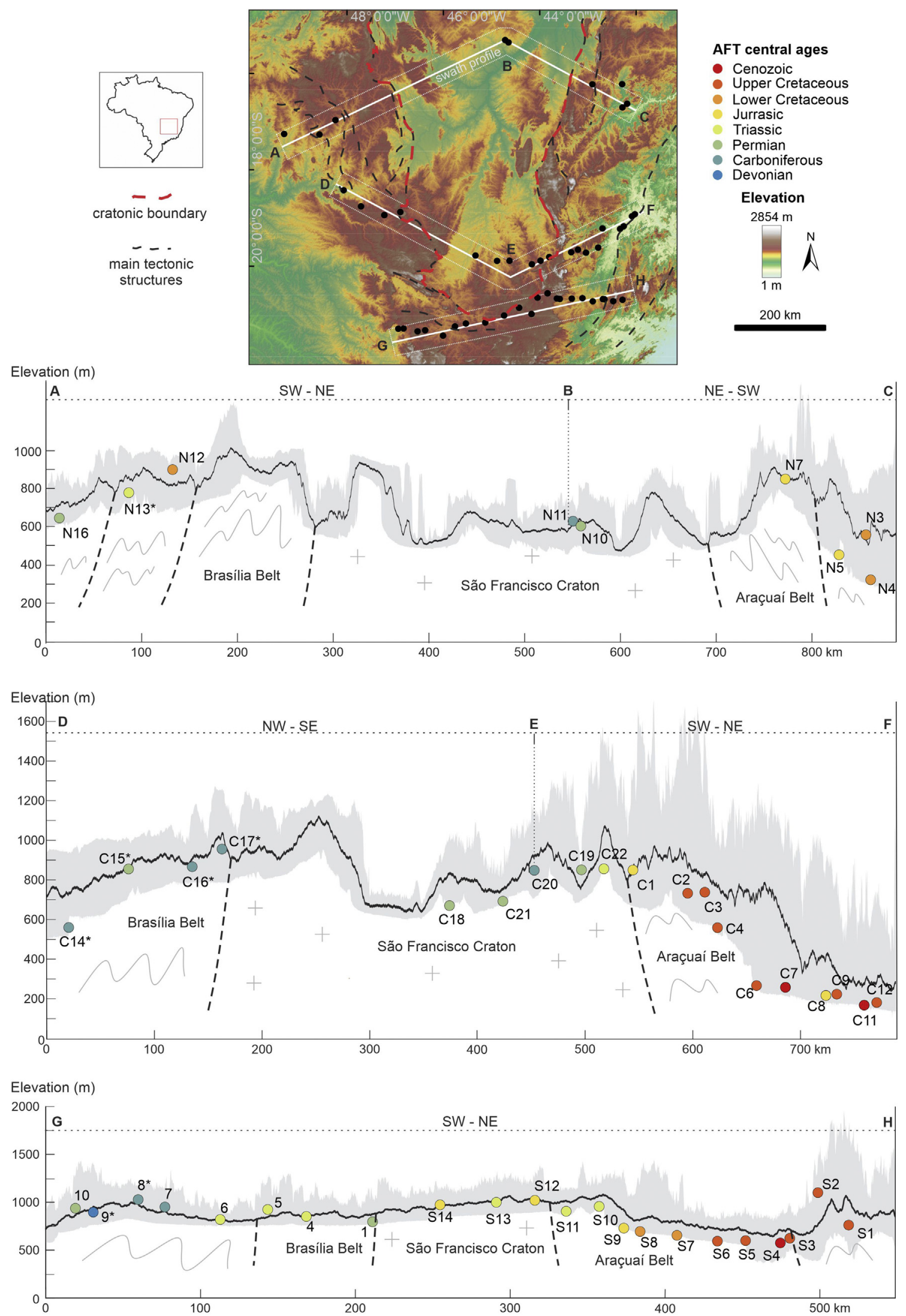

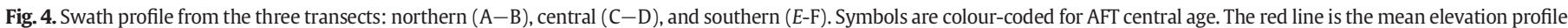

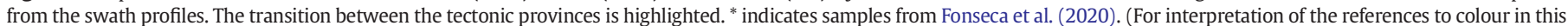
figure legend, the reader is referred to the web version of this article.) 


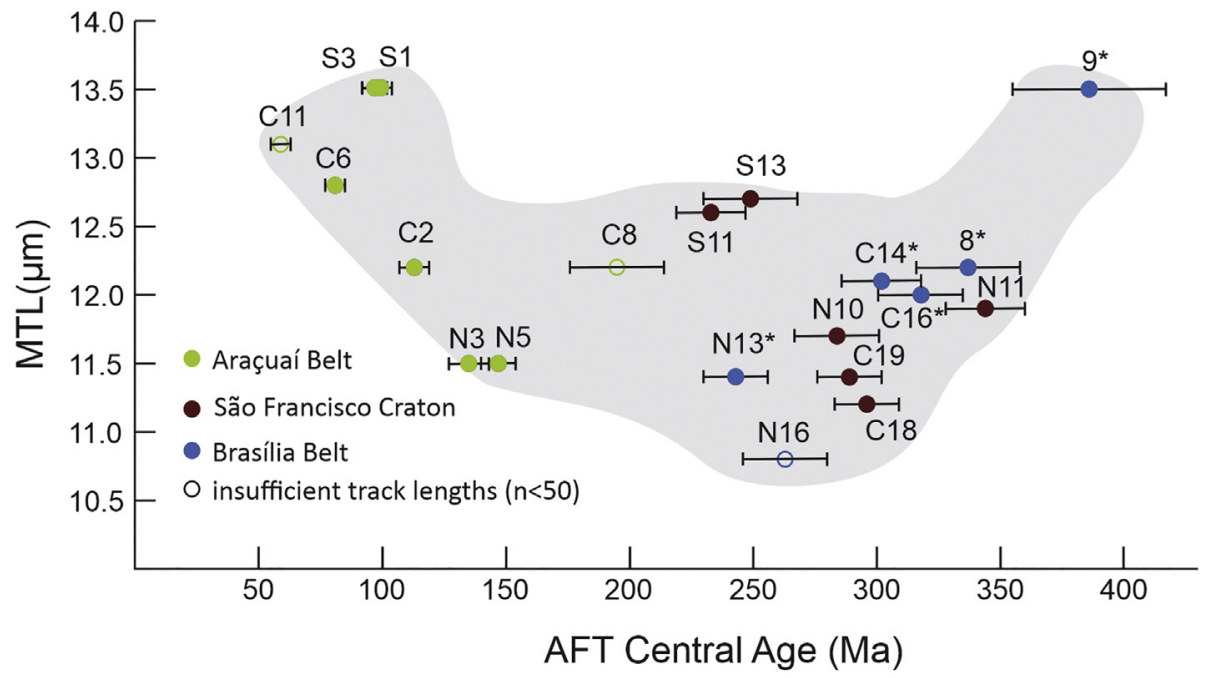

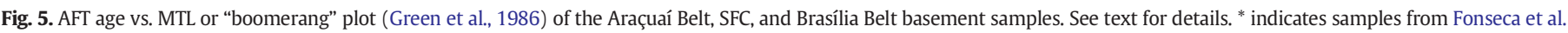
(2020). (For interpretation of the references to colour in this figure legend, the reader is referred to the web version of this article.)

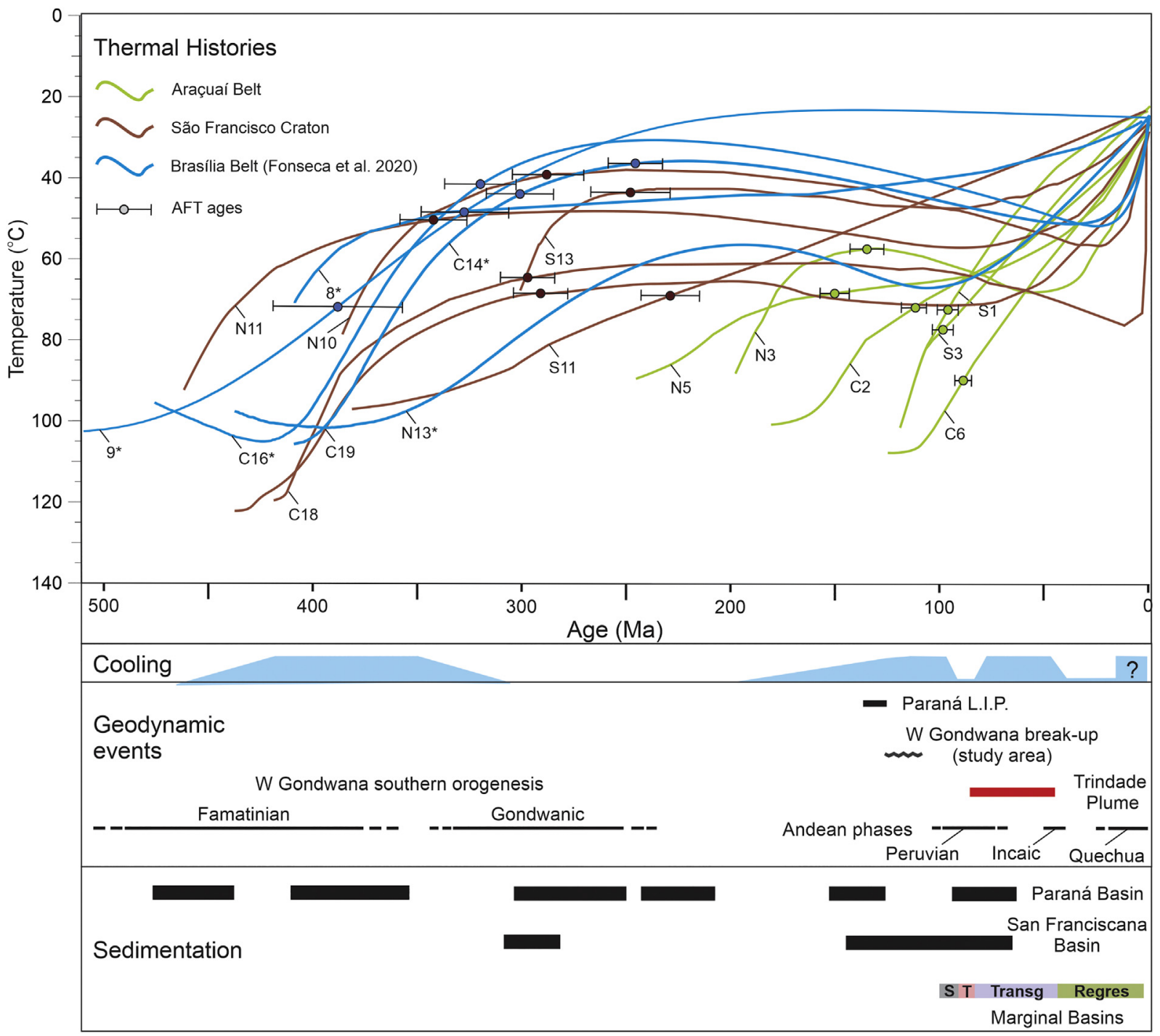

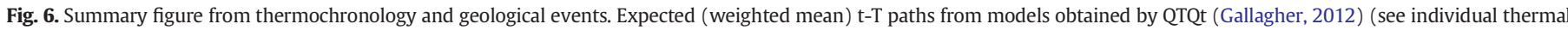

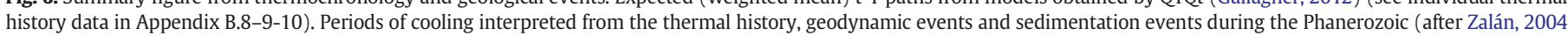
and Milani et al., 2007) are added. $\mathrm{S}=$ syn-rift and $\mathrm{T}=$ transitional phases in the marginal basins. 
Table. 1

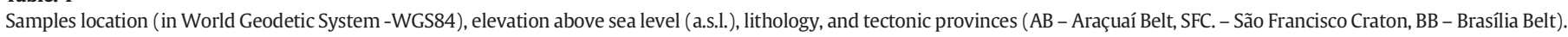

\begin{tabular}{|c|c|c|c|c|c|}
\hline Sample & $\mathrm{X}\left({ }^{\circ}\right)$ & $Y\left({ }^{\circ}\right)$ & Elevation (a.s.l.)(m) & Lithology & Province \\
\hline S1 & -42.2768 & -20.7010 & 718 & Banded granulite gneiss & $\mathrm{AB}$ \\
\hline $\mathrm{S} 2$ & -42.4713 & -20.7285 & 1192 & Medium grained piroxene granite & $\mathrm{AB}$ \\
\hline S3 & -42.6509 & -20.6900 & 651 & Medium grained piroxene granite & $\mathrm{AB}$ \\
\hline S4 & -42.6844 & -20.6869 & 614 & Biotite gneiss & $\mathrm{AB}$ \\
\hline S5 & -42.8959 & -20.7306 & 619 & Medium grained biotite gneiss & $\mathrm{AB}$ \\
\hline S6 & -43.1099 & -20.6665 & 615 & Fine grained biotite gneiss & $\mathrm{AB}$ \\
\hline S7 & -43.3320 & -20.6757 & 663 & Medium grained biotite gneiss & $\mathrm{AB}$ \\
\hline S8 & -43.5802 & -20.6839 & 722 & Fine grained biotite gneiss & $\mathrm{AB}$ \\
\hline S9 & -43.6389 & -20.6725 & 738 & Fine grained biotite gneiss & $\mathrm{AB}$ \\
\hline S10 & -43.8328 & -20.5669 & 926 & Coarse grained biotite granite & $\mathrm{AB}$ \\
\hline S12 & -44.1654 & -20.9957 & 1084 & Medium grained biotite granite & $\mathrm{AB}$ \\
\hline $\mathrm{C} 2$ & -43.3416 & -19.7112 & 720 & Coarse grained biotite gneiss & $\mathrm{AB}$ \\
\hline $\mathrm{C} 3$ & -43.2028 & -19.6612 & 735 & Biotite granite & $\mathrm{AB}$ \\
\hline C4 & -43.0367 & -19.7237 & 558 & Coarse grained biotite gneiss & $\mathrm{AB}$ \\
\hline C6 & -42.7839 & -19.6174 & 262 & Folded and sheared biotite gneiss & $\mathrm{AB}$ \\
\hline C7 & -42.6943 & -19.2180 & 242 & Biotite gneiss & $\mathrm{AB}$ \\
\hline C8 & -42.3276 & -19.2300 & 197 & Garnet bearing amphibolite & $\mathrm{AB}$ \\
\hline C9 & -42.2509 & -19.1723 & 208 & Fine grained biotite gneiss & $\mathrm{AB}$ \\
\hline C11 & -42.0818 & -18.9656 & 177 & Fine grained biotite gneiss & $\mathrm{AB}$ \\
\hline $\mathrm{C} 12$ & -42.0225 & -18.9231 & 188 & Fine grained biotite gneiss & $\mathrm{AB}$ \\
\hline N3 & -42.2801 & -16.7072 & 557 & Tourmaline bearing pegmatite & $\mathrm{AB}$ \\
\hline N4 & -42.1826 & -16.6299 & 310 & Biotite granite & $\mathrm{AB}$ \\
\hline N5 & -42.2846 & -16.2219 & 445 & Metagreywacke & $\mathrm{AB}$ \\
\hline N7 & -42.9033 & -16.2274 & 832 & Gneiss & $\mathrm{AB}$ \\
\hline S11 & -44.0412 & -20.6574 & 878 & Fine grained biotite gneiss & SFC \\
\hline S13 & -44.4227 & -20.8681 & 992 & Medium grained biotite granite & SFC \\
\hline S14 & -44.7343 & -21.0270 & 997 & Porphyritic basalt & SFC \\
\hline 1 & -45.1248 & -21.1736 & 786 & Migmatitic hornblende gneiss & SFC \\
\hline $\mathrm{C} 1$ & -43.7991 & -19.8133 & 855 & Migmatitic biotite gneiss & SFC \\
\hline $\mathrm{C} 22$ & -43.9794 & -19.8901 & 859 & Fine grained biotite gneiss & SFC \\
\hline C19 & -44.1580 & -19.9635 & 851 & Biotite gneiss & SFC \\
\hline $\mathrm{C} 20$ & -44.6217 & -19.8897 & 850 & Biotite granite & SFC \\
\hline $\mathrm{C} 21$ & -44.8803 & -19.8934 & 682 & Fine grained biotite gneiss & SFC \\
\hline C18 & -45.3290 & -19.7837 & 680 & Biotite bearing migmatite & SFC \\
\hline N11 & -44.7121 & -15.3076 & 620 & Granite & SFC \\
\hline N10 & -44.6414 & -15.3559 & 602 & Granite & SFC \\
\hline 4 & -45.5288 & -21.1933 & 805 & Medium grained horneblende granite & $\mathrm{BB}$ \\
\hline 5 & -45.7551 & -21.2543 & 893 & Biotite gneiss & BB \\
\hline 6 & -46.0035 & -21.4468 & 793 & Coarse grained piroxene gneiss & $\mathrm{BB}$ \\
\hline 7 & -46.3717 & -21.3339 & 883 & Coarse grained piroxene gneiss & $\mathrm{BB}$ \\
\hline $8^{\mathrm{a}}$ & -46.5302 & -21.3558 & 972 & Coarse grained piroxene gneiss & BB \\
\hline $9^{a}$ & -46.8217 & -21.2976 & 777 & Coarse grained piroxene gneiss & $\mathrm{BB}$ \\
\hline 10 & -46.9280 & -21.3034 & 791 & Granite & $\mathrm{BB}$ \\
\hline $\mathrm{C} 17^{\mathrm{a}}$ & -46.8887 & -18.8789 & 1061 & Metaconglomerate & $\mathrm{BB}$ \\
\hline $\mathrm{C} 16^{\mathrm{a}}$ & -47.2202 & -18.9419 & 884 & Biotite granite & $\mathrm{BB}$ \\
\hline $\mathrm{C} 15^{\mathrm{a}}$ & -47.7057 & -18.7589 & 856 & Biotite granite & $\mathrm{BB}$ \\
\hline $\mathrm{C} 14^{\mathrm{a}}$ & -48.0631 & -18.4268 & 577 & Biotite bearing migmatite & $\mathrm{BB}$ \\
\hline N12 & -48.2356 & -16.9667 & 898 & Garnet bearing gneiss & $\mathrm{BB}$ \\
\hline $\mathrm{N} 13^{\mathrm{a}}$ & -48.5663 & -17.2695 & 758 & Mylonitic granite & $\mathrm{BB}$ \\
\hline N16 & -49.3007 & -17.2581 & 631 & Mylonitic granite & BB \\
\hline
\end{tabular}

${ }^{a}$ Indicates samples from Fonseca et al. (2020).

\subsection{Araçuaí Orogen}

The twenty-four samples from the Araçuaí Orogen exhibit a wide range of AFT ages, from the late Triassic ( $210 \pm 10 \mathrm{Ma}$, Sample S10) to the early Paleogene ( $53 \pm 2 \mathrm{Ma}$, Sample C7). Ages from the Triassic to Early Cretaceous, i.e. pre- to syn-rift, are spatially situated closest to the SFC (samples S10, S8, S7 and C2; Figs. 3, 4) and in the northern transect ( samples N3, N4, N5, and N7; Figs. 3, 4). Ages from the Late Cretaceous to early Paleogene, i.e. post-rift, are spatially present in samples from the central and southern transects, located closer to the coast (Fig. A.1 in Appendix B). On the age-elevation plot (Fig. A.2 in Appendix B) the sample set from the Araçuaí Orogen describes a normal trend, in which the older AFT ages tend to be present in the samples collected at higher elevations. Samples C8 and S2 exhibit outlier's behavior, the former being "too old" for its elevation and the latter being "too young". Sample C8 was taken from a river valley at low elevation (197 m) and yields a Jurassic AFT age (195 $\pm 19 \mathrm{Ma}$ ). In the central profile (C-D, Fig. 4), it is the only sample with a pre-rift age in close proximity to samples yielding post-rift ages (Figs. 3, 4). Sample S2 is from a site at the highest elevation sampled and gives a Late Cretaceous AFT age $(81 \pm 7 \mathrm{Ma})$.

AFT length histograms from the Araçuaí Orogen samples are provided in Fig. A.3 in Appendix B. In six samples (S1, S3, C6, C2, N3 and $\mathrm{N} 5$ ) it was possible to measure more than 50 tracks to produce representative length-frequency histograms. Samples from the northern transect yield the shortest mean track lengths (MTL) $(11.5 \mu \mathrm{m}, \mathrm{N} 3$ and N5) and become longer to the south $(13.5 \mu \mathrm{m}, \mathrm{S} 1$ and S3). The histograms present a unimodal distribution. Standard deviation varies between 1.5 and $1.9 \mu \mathrm{m}$ and displays an apparent negative correlation with MTL (Fig. A.6 in Appendix B), i.e. the distribution becomes narrower with longer MTL. Skewness values are negative to nonskewed, ranging from -1.1 (Sample C6) to 0.0 (Sample N3), and describe negative correlation with MTL (Fig. A.7 in Appendix B), with two outliers (samples S1 and S3). MTL and AFT central ages establish a relationship as the half part of a boomerang trend (Fig. 5, Green et al., 1986). This relationship indicates a fast cooling through the 
Table. 2

Apatite fission track analysis results; $\mathrm{n}$ is the number of analyzed grains.

\begin{tabular}{|c|c|c|c|c|c|c|c|c|c|c|c|c|c|c|c|c|c|c|c|}
\hline & Sample & $\mathrm{n}$ & $\begin{array}{l}\rho_{\mathrm{s}} \\
( \pm 1 \sigma)\end{array}$ & $\mathrm{N}_{\mathrm{s}}$ & $\begin{array}{l}\rho_{\mathrm{i}} \\
( \pm 1 \sigma)\end{array}$ & $\mathrm{N}_{\mathrm{i}}$ & $\begin{array}{l}\rho_{\mathrm{d}} \\
( \pm 1 \sigma)\end{array}$ & $\mathrm{N}_{\mathrm{d}}$ & $\begin{array}{l}\mathrm{P} \\
\left(\chi^{2}\right)\end{array}$ & $\begin{array}{l}\text { AFT } \\
\text { central } \\
\text { age } \\
(\mathrm{Ma})\end{array}$ & $\begin{array}{l}1 \text { s.e. } \\
\text { (AFT } \\
\text { age) }\end{array}$ & $\mathrm{n}_{\mathrm{c}}$ & $\begin{array}{l}\text { MTL } \\
(\mu \mathrm{m})\end{array}$ & $\begin{array}{l}\sigma \\
(\mathrm{MTL}) \\
(\mu \mathrm{m})\end{array}$ & Skew-ness & $\begin{array}{l}D_{\text {par }} \\
(n)\end{array}$ & A & $\begin{array}{l}U_{\text {sample }} \\
(\mathrm{ppm})\end{array}$ & $\begin{array}{l}1 \text { s.e. } \\
\text { (U } U_{\text {sample })}\end{array}$ \\
\hline \multirow[t]{24}{*}{$\begin{array}{c}\text { Araçuaí } \\
\text { Belt }\end{array}$} & S1 & 20 & $\begin{array}{l}8.051 \\
(0.232)\end{array}$ & 1200 & $\begin{array}{l}5.859 \\
(0.232)\end{array}$ & 893 & $\begin{array}{l}5.774 \\
(0.075)\end{array}$ & 5999 & 0.25 & 98 & 6 & 81 & 13.5 & 1.5 & -0.555 & $\begin{array}{l}1.79 \\
(104)\end{array}$ & $\mathrm{T}$ & 13.3 & 0.7 \\
\hline & $\mathrm{S} 2$ & 11 & $\begin{array}{l}3.718 \\
(0.198)\end{array}$ & 350 & $\begin{array}{l}3.455 \\
(0.191)\end{array}$ & 327 & $\begin{array}{l}4.826 \\
(0.098)\end{array}$ & 2413 & 0.61 & 81 & 7 & $\mathrm{x}$ & $\mathrm{x}$ & $\mathrm{x}$ & $\mathrm{x}$ & $\begin{array}{l}1.69 \\
(153)\end{array}$ & A & 9.4 & 0.6 \\
\hline & S3 & 25 & $\begin{array}{l}8.512 \\
(0.201)\end{array}$ & 1789 & $\begin{array}{l}6.338 \\
(0.172)\end{array}$ & 1354 & $\begin{array}{l}5.716 \\
(0.073)\end{array}$ & 6054 & 0.43 & 97 & 5 & 87 & 13.5 & 1.7 & -0.533 & $\begin{array}{l}1.99 \\
(128)\end{array}$ & $\mathrm{T}$ & 14.6 & 0.7 \\
\hline & $S 4^{\mathrm{C}}$ & 9 & $\begin{array}{l}5.691 \\
(0.361)\end{array}$ & 249 & $\begin{array}{l}5.827 \\
(0.361)\end{array}$ & 261 & $\begin{array}{l}4.484 \\
(0.094)\end{array}$ & 2255 & 0.04 & 61 & 6 & $\mathrm{x}$ & $\mathrm{x}$ & $\mathrm{x}$ & $\mathrm{x}$ & $\mathrm{x}$ & $\mathrm{S}$ & 17.1 & 1.3 \\
\hline & S5 & 25 & $\begin{array}{l}5.464 \\
(0.149)\end{array}$ & 1339 & $\begin{array}{l}4.498 \\
(0.135)\end{array}$ & 1105 & $\begin{array}{l}4.750 \\
(0.097)\end{array}$ & 2375 & 0.18 & 90 & 5 & $\mathrm{x}$ & $\mathrm{x}$ & $\mathrm{x}$ & $\mathrm{x}$ & $\begin{array}{l}1.37 \\
(76)\end{array}$ & A & 12.4 & 0.6 \\
\hline & S6 & 22 & $\begin{array}{l}2.903 \\
(0.127)\end{array}$ & 524 & $\begin{array}{l}2.580 \\
(0.119)\end{array}$ & 471 & $\begin{array}{l}4.736 \\
(0.097)\end{array}$ & 2368 & 0.98 & 82 & 6 & $\mathrm{x}$ & $\mathrm{x}$ & $\mathrm{x}$ & $\mathrm{x}$ & $\mathrm{x}$ & A & 7.2 & 0.4 \\
\hline & S7 & 20 & $\begin{array}{l}27.528 \\
(0.506)\end{array}$ & 2963 & $\begin{array}{l}14.796 \\
(0.369)\end{array}$ & 1606 & $\begin{array}{l}4.722 \\
(0.097)\end{array}$ & 2361 & 0.44 & 135 & 6 & $\mathrm{x}$ & $\mathrm{x}$ & $\mathrm{x}$ & $\mathrm{x}$ & $\begin{array}{l}1.95 \\
(154)\end{array}$ & A & 41.2 & 2.0 \\
\hline & S8 & 18 & $\begin{array}{l}3.541 \\
(0.211)\end{array}$ & 281 & $\begin{array}{l}1.733 \\
(0.144)\end{array}$ & 144 & $\begin{array}{l}4.708 \\
(0.097)\end{array}$ & 2354 & 0.97 & 143 & 8 & $\mathrm{x}$ & $\mathrm{x}$ & $\mathrm{x}$ & $\mathrm{x}$ & $\mathrm{x}$ & A & 4.8 & 0.4 \\
\hline & S9 & 18 & $\begin{array}{l}22.570 \\
(0.696)\end{array}$ & 1052 & $\begin{array}{l}9.913 \\
(0.457)\end{array}$ & 470 & $\begin{array}{l}4.695 \\
(0.097)\end{array}$ & 2347 & 0.72 & 163 & 10 & $\mathrm{x}$ & $\mathrm{x}$ & $\mathrm{x}$ & $\mathrm{x}$ & $\begin{array}{l}1.79 \\
(183)\end{array}$ & A & 27.7 & 1.7 \\
\hline & S10 & 20 & $\begin{array}{l}34.751 \\
(0.704)\end{array}$ & 2434 & $\begin{array}{l}12.023 \\
(0.416)\end{array}$ & 836 & $\begin{array}{l}4.680 \\
(0.097)\end{array}$ & 2340 & 0.37 & 210 & 10 & $\mathrm{x}$ & $\mathrm{x}$ & $\mathrm{x}$ & $\mathrm{x}$ & $\begin{array}{l}1.85 \\
(188)\end{array}$ & A & 33.7 & 1.8 \\
\hline & S12 & 20 & $\begin{array}{l}7.527 \\
(0.303)\end{array}$ & 616 & $\begin{array}{l}3.587 \\
(0.214)\end{array}$ & 280 & $\begin{array}{l}4.650 \\
(0.096)\end{array}$ & 2325 & 0.12 & 158 & 14 & $x$ & $\mathrm{x}$ & $\mathrm{x}$ & $\mathrm{x}$ & $\mathrm{x}$ & A & 10.1 & 0.7 \\
\hline & $\mathrm{C} 2$ & 22 & $\begin{array}{l}8.757 \\
(0.244)\end{array}$ & 1238 & $\begin{array}{l}5.790 \\
(0.201)\end{array}$ & 831 & $\begin{array}{l}4.855 \\
(0.099)\end{array}$ & 2427 & 0.57 & 113 & 6 & 54 & 12.2 & 1.6 & -0.317 & $\begin{array}{l}1.74 \\
(205)\end{array}$ & A & 15.7 & 0.8 \\
\hline & $C 3^{c}$ & 16 & $\begin{array}{l}4.164 \\
(0.267)\end{array}$ & 243 & $\begin{array}{l}3.160 \\
(0.230)\end{array}$ & 189 & $\begin{array}{l}4.475 \\
(0.095)\end{array}$ & 2241 & 0.00 & 82 & 14 & $\mathrm{x}$ & $\mathrm{x}$ & $\mathrm{x}$ & $\mathrm{x}$ & $\mathrm{x}$ & $\mathrm{S}$ & 9.3 & 0.8 \\
\hline & C4 & 20 & $\begin{array}{l}5.980 \\
(0.173)\end{array}$ & 1196 & $\begin{array}{l}5.100 \\
(0.160)\end{array}$ & 1020 & $\begin{array}{l}4.732 \\
(0.097)\end{array}$ & 2366 & 0.29 & 86 & 4 & $x$ & $\mathrm{x}$ & $\mathrm{x}$ & $x$ & $\begin{array}{l}1.74 \\
(152)\end{array}$ & A & 14.2 & 0.7 \\
\hline & C6 & 21 & $\begin{array}{l}14.375 \\
(0.263)\end{array}$ & 2976 & $\begin{array}{l}13.189 \\
(0.252)\end{array}$ & 2736 & $\begin{array}{l}4.723 \\
(0.097)\end{array}$ & 2365 & 0.06 & 81 & 4 & 88 & 12.8 & 1.6 & -0.981 & $\begin{array}{l}2.01 \\
(200)\end{array}$ & A & 36.7 & 1.7 \\
\hline & $\mathrm{C7}$ & 21 & $\begin{array}{l}8.967 \\
(0.206)\end{array}$ & 1888 & $\begin{array}{l}10.234 \\
(0.221)\end{array}$ & 2154 & $\begin{array}{l}3.841 \\
(0.088)\end{array}$ & 1920 & 0.17 & 53 & 2 & $\mathrm{x}$ & $\mathrm{x}$ & $\mathrm{x}$ & $\mathrm{x}$ & $\mathrm{x}$ & A & 35.0 & 1.7 \\
\hline & $\mathrm{C} 8^{\mathrm{b}}$ & 8 & $\begin{array}{l}6.841 \\
(0.326)\end{array}$ & 440 & $\begin{array}{l}2.536 \\
(0.197)\end{array}$ & 165 & $\begin{array}{l}4.727 \\
(0.097)\end{array}$ & 2363 & 0.44 & 195 & 19 & $29^{b}$ & $12.2^{\mathrm{b}}$ & $1.5^{\mathrm{b}}$ & 0.011 & $\begin{array}{l}1.71 \\
(122)\end{array}$ & A & 7.0 & 0.6 \\
\hline & C9 & 20 & $\begin{array}{l}3.508 \\
(0.136)\end{array}$ & 661 & $\begin{array}{l}3.774 \\
(0.142)\end{array}$ & 709 & $\begin{array}{l}4.724 \\
(0.097)\end{array}$ & 2362 & 0.71 & 69 & 4 & $\mathrm{x}$ & $\mathrm{x}$ & $\mathrm{x}$ & $\mathrm{x}$ & $\begin{array}{l}1.68 \\
(139)\end{array}$ & A & 10.5 & 0.6 \\
\hline & $\mathrm{C} 11^{\mathrm{b}}$ & 20 & $\begin{array}{l}3.872 \\
(0.143)\end{array}$ & 730 & $\begin{array}{l}4.792 \\
(0.159)\end{array}$ & 905 & $\begin{array}{l}4.722 \\
(0.097)\end{array}$ & 2361 & 0.23 & 59 & 4 & $42^{\mathrm{b}}$ & $13.1^{b}$ & $1.3^{\mathrm{b}}$ & -0.643 & $\begin{array}{l}1.83 \\
(116)\end{array}$ & A & 13.3 & 0.7 \\
\hline & C12 & 20 & $\begin{array}{l}3.592 \\
(0.137)\end{array}$ & 689 & $\begin{array}{l}3.997 \\
(0.145)\end{array}$ & 762 & $\begin{array}{l}4.719 \\
(0.097)\end{array}$ & 2360 & 0.36 & 67 & 4 & $x$ & $\mathrm{x}$ & $\mathrm{x}$ & $\mathrm{x}$ & $\begin{array}{l}2.04 \\
(115)\end{array}$ & A & 11.1 & 0.6 \\
\hline & N3 & 7 & $\begin{array}{l}31.484 \\
(0.912)\end{array}$ & 1192 & $\begin{array}{l}16.951 \\
(0.670)\end{array}$ & 642 & $\begin{array}{l}4.684 \\
(0.097)\end{array}$ & 2342 & 0.25 & 135 & 8 & 69 & 11.5 & 1.8 & 0.017 & $\begin{array}{l}1.95 \\
(239)\end{array}$ & A & 47.5 & 2.7 \\
\hline & N4 & 23 & $\begin{array}{l}3.906 \\
(0.149)\end{array}$ & 687 & $\begin{array}{l}2.745 \\
(0.127)\end{array}$ & 469 & $\begin{array}{l}4.681 \\
(0.097)\end{array}$ & 2341 & 0.09 & 107 & 8 & $\mathrm{x}$ & $\mathrm{x}$ & $\mathrm{x}$ & $\mathrm{x}$ & $\begin{array}{l}1.84 \\
(154)\end{array}$ & A & 7.7 & 0.5 \\
\hline & N5 & 23 & $\begin{array}{l}12.709 \\
(0.270)\end{array}$ & 2214 & $\begin{array}{l}6.293 \\
(0.190)\end{array}$ & 1093 & $\begin{array}{l}4.679 \\
(0.097)\end{array}$ & 2340 & 0.21 & 147 & 7 & 50 & 11.5 & 1.9 & -0.374 & $\begin{array}{l}1.78 \\
(172)\end{array}$ & A & 17.7 & 0.9 \\
\hline & N7 & 2 & $\begin{array}{l}7.016 \\
(0.973)\end{array}$ & 52 & $\begin{array}{l}3.071 \\
(0.627)\end{array}$ & 24 & $\begin{array}{l}4.664 \\
(0.097)\end{array}$ & 2332 & 0.32 & 157 & 40 & $\mathrm{x}$ & $\mathrm{x}$ & $\mathrm{x}$ & $\mathrm{x}$ & $\mathrm{x}$ & A & 8.6 & 1.8 \\
\hline \multirow{12}{*}{$\begin{array}{l}\text { São } \\
\text { Francisco } \\
\text { Craton }\end{array}$} & S11 & 25 & $\begin{array}{l}12.780 \\
(0.271)\end{array}$ & 2231 & $\begin{array}{l}3.920 \\
(0.149)\end{array}$ & 696 & $\begin{array}{l}5.800 \\
(0.074)\end{array}$ & 6126 & 0.43 & 233 & 14 & 56 & 12.6 & 1.9 & -0.317 & $\begin{array}{l}1.66 \\
(142)\end{array}$ & $\mathrm{T}$ & 8.9 & 0.5 \\
\hline & S13 & 21 & $\begin{array}{l}18.893 \\
(0.378)\end{array}$ & 2500 & $\begin{array}{l}5.624 \\
(0.208)\end{array}$ & 728 & $\begin{array}{l}5.795 \\
(0.074)\end{array}$ & 6148 & 0.12 & 249 & 19 & 91 & 12.7 & 1.3 & 0.276 & $\begin{array}{l}1.75 \\
(197)\end{array}$ & $\mathrm{T}$ & 12.7 & 0.7 \\
\hline & S14 & 20 & $\begin{array}{l}7.123 \\
(0.264)\end{array}$ & 2018 & $\begin{array}{l}2.822 \\
(0.165)\end{array}$ & 291 & $\begin{array}{l}4.514 \\
(0.094)\end{array}$ & 2301 & 0.77 & 160 & 12 & $\mathrm{x}$ & $\mathrm{x}$ & $\mathrm{x}$ & $\mathrm{x}$ & $\mathrm{x}$ & $\mathrm{S}$ & 8.2 & 0.6 \\
\hline & 1 & 23 & $\begin{array}{l}26.051 \\
(0.483)\end{array}$ & 2908 & $\begin{array}{l}7.843 \\
(0.268)\end{array}$ & 860 & $\begin{array}{l}4.954 \\
(0.100)\end{array}$ & 2477 & 0.22 & 257 & 13 & $\mathrm{x}$ & $\mathrm{x}$ & $\mathrm{x}$ & $\mathrm{x}$ & $\begin{array}{l}2.04 \\
(115)\end{array}$ & A & 20.8 & 1.1 \\
\hline & $\mathrm{C} 1$ & 25 & $\begin{array}{l}10.173 \\
(0.253)\end{array}$ & 1615 & $\begin{array}{l}4.520 \\
(0.167)\end{array}$ & 719 & $\begin{array}{l}4.870 \\
(0.099)\end{array}$ & 2435 & 0.53 & 169 & 9 & $\mathrm{x}$ & $\mathrm{x}$ & $\mathrm{x}$ & $\mathrm{x}$ & $\begin{array}{l}1.36 \\
(200)\end{array}$ & A & 12.2 & 0.7 \\
\hline & $\mathrm{C} 22$ & 20 & $\begin{array}{l}30.203 \\
(0.535)\end{array}$ & 3191 & $\begin{array}{l}9.420 \\
(0.297)\end{array}$ & 999 & $\begin{array}{l}4.687 \\
(0.097)\end{array}$ & 2343 & 0.26 & 231 & 11 & $\mathrm{x}$ & $\mathrm{x}$ & $\mathrm{x}$ & $\mathrm{x}$ & $\begin{array}{l}1.90 \\
(215)\end{array}$ & A & 26.4 & 1.4 \\
\hline & C19 & 22 & $\begin{array}{l}21.493 \\
(0.345)\end{array}$ & 3881 & $\begin{array}{l}5.361 \\
(0.172)\end{array}$ & 969 & $\begin{array}{l}4.694 \\
(0.097)\end{array}$ & 2347 & 0.34 & 289 & 13 & 100 & 11.4 & 1.8 & -0.052 & $\begin{array}{l}1.86 \\
(124)\end{array}$ & A & 15.0 & 0.8 \\
\hline & $\mathrm{C} 20$ & 20 & $\begin{array}{l}25.769 \\
(0.438)\end{array}$ & 3467 & $\begin{array}{l}5.995 \\
(0.214)\end{array}$ & 785 & $\begin{array}{l}4.691 \\
(0.097)\end{array}$ & 2346 & 0.09 & 316 & 17 & $\mathrm{x}$ & $\mathrm{x}$ & $\mathrm{x}$ & $\mathrm{x}$ & $\begin{array}{l}1.98 \\
(188)\end{array}$ & A & 16.8 & 0.9 \\
\hline & $\mathrm{C} 21$ & 22 & $\begin{array}{l}17.477 \\
(0.304)\end{array}$ & 3310 & $\begin{array}{l}4.799 \\
(0.160)\end{array}$ & 904 & $\begin{array}{l}4.689 \\
(0.097)\end{array}$ & 2344 & 0.29 & 262 & 13 & $\mathrm{x}$ & $\mathrm{x}$ & $\mathrm{x}$ & $\mathrm{x}$ & $\begin{array}{l}1.89 \\
(146)\end{array}$ & A & 13.4 & 0.7 \\
\hline & C18 & 22 & $\begin{array}{l}28.598 \\
(0.363)\end{array}$ & 6220 & $\begin{array}{l}7.008 \\
(0.179)\end{array}$ & 1527 & $\begin{array}{l}4.696 \\
(0.097)\end{array}$ & 2348 & 0.07 & 296 & 13 & 100 & 11.2 & 1.4 & 0.050 & $\begin{array}{l}1.98 \\
(221)\end{array}$ & A & 19.6 & 1.0 \\
\hline & N11 & 21 & $\begin{array}{l}35.810 \\
(0.560)\end{array}$ & 4083 & $\begin{array}{l}7.523 \\
(0.259)\end{array}$ & 844 & $\begin{array}{l}4.656 \\
(0.097)\end{array}$ & 2328 & 0.59 & 344 & 16 & 87 & 11.9 & 1.6 & 0.070 & $\begin{array}{l}2.02 \\
(237)\end{array}$ & A & 21.2 & 1.1 \\
\hline & N10 & 20 & 20.383 & 2455 & 5.054 & 614 & 4.659 & 2329 & 0.14 & 284 & 17 & 100 & 11.7 & 1.3 & -0.146 & 1.71 & A & 14.2 & 0.8 \\
\hline
\end{tabular}


Table. 2 (continued)

\begin{tabular}{|c|c|c|c|c|c|c|c|c|c|c|c|c|c|c|c|c|c|c|c|}
\hline & Sample & $n$ & $\begin{array}{l}\rho_{\mathrm{s}} \\
( \pm 1 \sigma)\end{array}$ & $\mathrm{N}_{\mathrm{s}}$ & $\begin{array}{l}\rho_{\mathrm{i}} \\
( \pm 1 \sigma)\end{array}$ & $\mathrm{N}_{\mathrm{i}}$ & $\begin{array}{l}\rho_{\mathrm{d}} \\
( \pm 1 \sigma)\end{array}$ & $\mathrm{N}_{\mathrm{d}}$ & $\begin{array}{l}\mathrm{P} \\
\left(\chi^{2}\right)\end{array}$ & $\begin{array}{l}\text { AFT } \\
\text { central } \\
\text { age } \\
\text { (Ma) }\end{array}$ & $\begin{array}{l}1 \text { s.e. } \\
\text { (AFT } \\
\text { age) }\end{array}$ & $\mathrm{n}_{\mathrm{c}}$ & $\begin{array}{l}\text { MTL } \\
(\mu \mathrm{m})\end{array}$ & $\begin{array}{l}\sigma \\
(\mathrm{MTL}) \\
(\mu \mathrm{m})\end{array}$ & Skew-ness & $\begin{array}{l}D_{\text {par }} \\
(\mathrm{n})\end{array}$ & A & $\begin{array}{l}\mathrm{U}_{\text {sample }} \\
\text { (ppm) }\end{array}$ & $\begin{array}{l}1 \text { s.e. } \\
\text { (U } U_{\text {sample) }}\end{array}$ \\
\hline \multirow{15}{*}{$\begin{array}{c}\text { Brasília } \\
\text { belt }\end{array}$} & & & $(0.411)$ & & $(0.204)$ & & $(0.097)$ & & & & & & & & & (243) & & & \\
\hline & 4 & 21 & $\begin{array}{l}25.332 \\
(0.529)\end{array}$ & 2291 & $\begin{array}{l}7.866 \\
(0.292)\end{array}$ & 726 & $\begin{array}{l}4.941 \\
(0.099)\end{array}$ & 2470 & 0.86 & 240 & 12 & $\mathrm{x}$ & $\mathrm{x}$ & $\mathrm{x}$ & $\mathrm{x}$ & $\begin{array}{l}2.05 \\
(191)\end{array}$ & A & 20.9 & 1.2 \\
\hline & 5 & 20 & $\begin{array}{l}12.159 \\
(0.393)\end{array}$ & 959 & $\begin{array}{l}3.659 \\
(0.214)\end{array}$ & 293 & $\begin{array}{l}4.465 \\
(0.095)\end{array}$ & 2225 & 0.62 & 206 & 15 & $\mathrm{x}$ & $\mathrm{x}$ & $\mathrm{x}$ & $\mathrm{x}$ & $\mathrm{x}$ & S & 10.8 & 0.8 \\
\hline & 6 & 20 & $\begin{array}{l}9.860 \\
(0.212)\end{array}$ & 2167 & $\begin{array}{l}3.205 \\
(0.121)\end{array}$ & 702 & $\begin{array}{l}4.926 \\
(0.099)\end{array}$ & 2463 & 0.09 & 234 & 14 & $\mathrm{x}$ & $\mathrm{x}$ & $\mathrm{x}$ & $\mathrm{x}$ & $\begin{array}{l}1.67 \\
(120)\end{array}$ & A & 8.5 & 0.5 \\
\hline & 7 & 21 & $\begin{array}{l}12.700 \\
(0.355)\end{array}$ & 1282 & $\begin{array}{l}2.791 \\
(0.164)\end{array}$ & 291 & $\begin{array}{l}4.911 \\
(0.099)\end{array}$ & 2456 & 0.81 & 331 & 23 & $\mathrm{x}$ & $\mathrm{x}$ & $\mathrm{x}$ & $\mathrm{x}$ & $\begin{array}{l}1.69 \\
(155)\end{array}$ & A & 7.5 & 0.5 \\
\hline & $8^{a}$ & 25 & $\begin{array}{l}15.481 \\
(0.308)\end{array}$ & 2524 & $\begin{array}{l}3.250 \\
(0.140)\end{array}$ & 542 & $\begin{array}{l}5.818 \\
(0.075)\end{array}$ & 5957 & 0.20 & 337 & 21 & 80 & 12.2 & 1.3 & 0.063 & $\begin{array}{l}1.50 \\
(202)\end{array}$ & a & 7.3 & 0.4 \\
\hline & $9^{a}$ & 20 & $\begin{array}{l}5.780 \\
(0.170)\end{array}$ & 1156 & $\begin{array}{l}1.070 \\
(0.073)\end{array}$ & 214 & $\begin{array}{l}5.807 \\
(0.075)\end{array}$ & 5967 & 0.91 & 386 & 31 & 100 & 13.5 & 1.2 & -0.386 & $\begin{array}{l}1.70 \\
(105)\end{array}$ & a & 2.4 & 0.2 \\
\hline & 10 & 20 & $\begin{array}{l}35.992 \\
(0.760)\end{array}$ & 2241 & $\begin{array}{l}8.844 \\
(0.380)\end{array}$ & 543 & $\begin{array}{l}4.533 \\
(0.094)\end{array}$ & 2331 & 0.59 & 261 & 19 & $\mathrm{x}$ & $\mathrm{x}$ & $\mathrm{x}$ & $\mathrm{x}$ & $\mathrm{x}$ & S & 25.6 & 1.5 \\
\hline & $\mathrm{C} 17^{\mathrm{a}}$ & 7 & $\begin{array}{l}14.702 \\
(0.798)\end{array}$ & 340 & $\begin{array}{l}3.203 \\
(0.367)\end{array}$ & 76 & $\begin{array}{l}5.769 \\
(0.106)\end{array}$ & 2940 & 0.24 & 314 & 41 & $\mathrm{x}$ & $\mathrm{x}$ & $\mathrm{x}$ & $\mathrm{x}$ & $\mathrm{x}$ & a & 7.3 & 0.9 \\
\hline & $\mathrm{C} 16^{\mathrm{a}}$ & 20 & $\begin{array}{l}28.350 \\
(0.493)\end{array}$ & 3302 & $\begin{array}{l}6.230 \\
(0.231)\end{array}$ & 729 & $\begin{array}{l}5.766 \\
(0.106)\end{array}$ & 2954 & 0.36 & 318 & 17 & 105 & 12.0 & 1.2 & -0.609 & $\begin{array}{l}1.91 \\
(169)\end{array}$ & a & 14.2 & 0.8 \\
\hline & $\mathrm{C} 15^{\mathrm{a}}$ & 3 & $\begin{array}{l}28.298 \\
(1.636)\end{array}$ & 299 & $\begin{array}{l}7.245 \\
(0.820)\end{array}$ & 78 & $\begin{array}{l}5.766 \\
(0.106)\end{array}$ & 2954 & 0.25 & 269 & 37 & $\mathrm{x}$ & $\mathrm{x}$ & $\mathrm{x}$ & $\mathrm{x}$ & $\mathrm{x}$ & a & 16.5 & 2.0 \\
\hline & $\mathrm{C} 14^{\mathrm{a}}$ & 20 & $\begin{array}{l}23.620 \\
(0.473)\end{array}$ & 2491 & $\begin{array}{l}5.419 \\
(0.225)\end{array}$ & 579 & $\begin{array}{l}5.764 \\
(0.106)\end{array}$ & 2953 & 0.48 & 302 & 16 & 105 & 12.1 & 1.2 & -0.387 & $\begin{array}{l}1.95 \\
(281)\end{array}$ & a & 12.3 & 0.7 \\
\hline & N12 & 9 & $\begin{array}{l}4.486 \\
(0.382)\end{array}$ & 138 & $\begin{array}{l}2.987 \\
(0.306)\end{array}$ & 95 & $\begin{array}{l}4.654 \\
(0.096)\end{array}$ & 2327 & 0.48 & 106 & 15 & $\mathrm{x}$ & $x$ & $\mathrm{x}$ & $\mathrm{x}$ & $\begin{array}{l}1.79 \\
(117)\end{array}$ & A & 8.4 & 0.9 \\
\hline & $\mathrm{N} 13^{\mathrm{a}}$ & 20 & $\begin{array}{l}30.056 \\
(0.679)\end{array}$ & 2024 & $\begin{array}{l}9.005 \\
(0.370)\end{array}$ & 592 & $\begin{array}{l}5.827 \\
(0.107)\end{array}$ & 2985 & 0.50 & 243 & 13 & 100 & 11.4 & 1.8 & 0.061 & $\begin{array}{l}2.07 \\
(178)\end{array}$ & a & 20.3 & 1.2 \\
\hline & N16 ${ }^{\mathrm{b}}$ & 23 & $\begin{array}{l}11.606 \\
(0.308)\end{array}$ & 1423 & $\begin{array}{l}3.261 \\
(0.166)\end{array}$ & 387 & $\begin{array}{l}4.646 \\
(0.096)\end{array}$ & 2323 & 0.23 & 263 & 17 & $24^{\mathrm{b}}$ & $10.8^{\mathrm{b}}$ & $1.9^{\mathrm{b}}$ & 0.310 & $\begin{array}{l}1.73 \\
(133)\end{array}$ & A & 9.2 & 0.6 \\
\hline
\end{tabular}

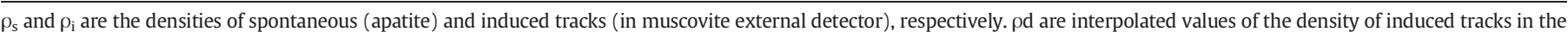

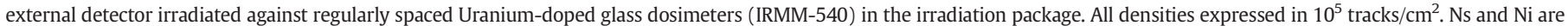

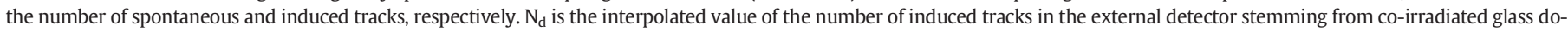

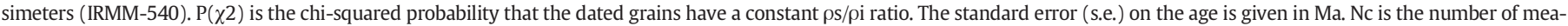

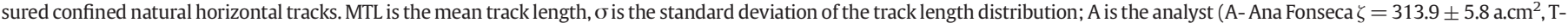

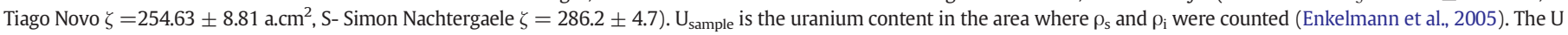
content standard error (s.e.) is given in $\mathrm{ppm}$.

a Indicates samples from Fonseca et al. (2020).

b Indicates lengths analysis with insufficient measurements to perform inverse thermal history modeling.

c Indicates samples that failed in the $\mathrm{P}(\chi 2)$ test.

APAZ for samples S1, S3, C11 and C6 (longer MTL), while samples C2, C8, N3 and N5 (shorter MTL) had a protracted residence in the APAZ.

Thermal history modeling was performed for six samples from the Araçuaí Orogen (samples S1, S3, C2, C6, N3 and N5; Fig. A.8 in Appendix $B)$. In the southern and central transects, the expected T-t path of samples S1, S3 and C6 display a similar time-temperature path. For these samples the models show a cooling through the $100^{\circ} \mathrm{C}$ isotherm around $120 \mathrm{Ma}$, followed by a quasi linear, protracted cooling since ca. $110 \mathrm{Ma}$. The expected T-t path of sample $\mathrm{C} 2$ displays a residence in the temperature window between $\sim 100{ }^{\circ} \mathrm{C}$ to $80{ }^{\circ} \mathrm{C}$ between ca. 170 to $140 \mathrm{Ma}$ when it also begins to transfer into a quasi linear, protracted cooling until surface temperature is reached. In the northern transect, the expected T-t path of samples N3 and N5 show a cooling phase during the Triassic to Early Jurassic (N5 until ca. $200 \mathrm{Ma}$ and N3 until ca. $130 \mathrm{Ma}$ ) followed by thermal quiescence (or modest reheating) before a subsequent fast cooling since ca. 50 Ma.

\subsection{São Francisco Craton}

The twelve samples from the SFC exhibit pre-rift AFT central ages ranging from early Carboniferous ( $344 \pm 16 \mathrm{Ma}$, Sample N11) to Late Jurassic (160 $\pm 13 \mathrm{Ma}$, Sample S14). The majority of the samples close to the boundary with the orogenic belts (S11, S13, S14, C1 and C22) present Triassic to Jurassic ages. In the cratonic interior, samples yield ages from Carboniferous to Permian (C18, C21, C20, C19, N10 and
N11). These sample were collected from 602 to $997 \mathrm{~m}$ elevation above sea level (a.s.l.) and their altitudes show no clear relationship with AFT central age (Fig. A.2 in Appendix B).

AFT length histograms from the SFC samples are provided in Fig. A.4 in Appendix B. In six samples (S11, S13, C18, C19, N10 and N11) it was possible to produce representative length-frequency histograms. Samples from the southern transect display the longest MTL (12.7 and $12.6 \mu \mathrm{m}$, samples S13 and S11, respectively) whereas in the others the MTLs range from $11.2 \mu \mathrm{m}$ (Sample C18) to $11.9 \mu \mathrm{m}$ (Sample N11). The distributions are unimodal with standard deviations between 1.3 and $1.9 \mu \mathrm{m}$, showing no correlation with MTL (Fig. A.6 in Appendix B). The histograms are quasi symmetric with skewness varying between -0.3 (Sample S11) to 0.3 (Sample S13). Skewness and MTL describe a negative correlation (Fig. A.7 in Appendix B), although one outlier is present (Sample S13). The MTL versus AFT central age plot suggests a half part of a boomerang trend (Fig. 5, Green et al., 1986), however the longest tracks of the SFC samples are relatively short compared to the higher MTLs of the analyzed orogenic belts.

Thermal history modeling was performed on data from six samples from the SFC (samples S11, S13, C18, C19, N10 and N11). Except for the expected model of sample S11, which predicts a constant slow rate of cooling up to surfaces temperatures since ca. $300 \mathrm{Ma}$, all expected T- $\mathrm{t}$ path of the samples display a relatively fast cooling during the Paleozoic (N11 until ca. $400 \mathrm{Ma}, \mathrm{C} 18$ until ca. $390 \mathrm{Ma} \mathrm{N10}$ and C19 until ca. $350 \mathrm{Ma}$ and S3 until ca. $290 \mathrm{Ma}$ ) followed by a long period of thermal stability 
(60 to $40{ }^{\circ} \mathrm{C}$ ) in the Mesozoic and afterward a new cooling pulse since ca. $50 \mathrm{Ma}$.

\subsection{Brasília Orogen}

The majority of the seven samples from Brasilia Orogen exhibit AFT ages ranging from the Triassic ( $206 \pm 15 \mathrm{Ma}$, Sample 5) to the Carboniferous (331 $\pm 23 \mathrm{Ma}$, Sample 7). Sample N12 presents a disparate Albian AFT age ( $106 \pm 15 \mathrm{Ma})$. Even though its grains counting passed in the Chi-squared test $\left(\mathrm{P}\left(\chi^{2}\right)=48 \%\right.$ ), only nine grains could be analyzed. This result thus needs to be regarded with carefulness. The ageelevation plot (Fig. A.2 in Appendix B) does not reveal any clear relationship between the variables.

\section{Discussion}

\subsection{Timing and spatial patterns inferred from thermochronology}

Low-temperature thermochronology has only seldomly been applied to basement rocks in the Brazilian interior, in particular not adjacent to our study area, thus the majority of our AFT data covers unexplored locality. Our new AFT data can be compared to a limited number of published AFT results (Amaral-Santos et al., 2019; Carmo, 2005; Doranti-Tiritan et al., 2014; Fonseca et al., 2020; Gallagher et al., 1994; Hackspacher et al., 2004; Jelinek et al., 2014; Martins-Ferreira et al., 2020; Van Ranst et al., 2020a) and apatite (U-Th)/He (AHe) data (Van Ranst et al., 2020a) from samples from some adjacent areas. Where they spatially connect, our AFT data are in agreement with these previous studies. Especially samples from close proximity, such as samples BR-30 and BR-1 from Van Ranst et al. (2020a) compared with samples from this study (C12 and S1, respectively) lie within expected statistical uncertainty. Samples from Fonseca et al. (2020) complete our dataset on the Brasília Orogen providing additional MTL information (Fig. A.5 in Appendix B), and presenting inversion timetemperature models (Fig. A.10 in Appendix B), therefore they are combined with our dataset for further discussion.

A clear distinction with respect to the tectonic province can readily be observed in the AFT data set presented here. In general, all AFT central ages range between $386 \pm 31 \mathrm{Ma}$ (sample 9 ) and $59 \pm 4 \mathrm{Ma}$ (Sample C11). AFT ages from the Araçuaí Orogen range from $210 \pm 10 \mathrm{Ma}$ to $59 \pm 4 \mathrm{Ma}$ (Table 2) while ages from the Brasília Orogen and the SFC are generally older, ranging between $386 \pm 31$ Ma to $160 \pm 12 \mathrm{Ma}$ (Table 2) with one outlier (Sample N12, $106 \pm 15 \mathrm{Ma}$ ). The intermediate ages between these two groups ( 160 to $210 \mathrm{Ma}$ ) predominate in the transition zone between the Araçuaí Orogen and the SFC (Figs. 3 and 4). The relationship between elevation and AFT central age is also distinct. While the Araçuaí Orogen samples show a normal trend, i.e. the older ages at higher elevation, the samples from the Brasília Orogen and the SFC display no clear correlation and suggest an exhumed APAZ signature (Fig. A.6 in Appendix B).

In the AFT samples from the Araçuaí Orogen, the MTL is longer $(13.5 \mu \mathrm{m})$ in samples with AFT central ages around $95 \mathrm{Ma}$ (sample S1 and S3) and the MTL tends to decrease (with increasing standard deviation) for samples with ages between $110 \mathrm{Ma}$ and $150 \mathrm{Ma}$ (Fig. 5). Hence, it is possible to suggest a rather rapid cooling around $95 \mathrm{Ma}$ (Green et al., 1986). In samples from the Brasília Orogen and the SFC, the longest MTL $(13.7 \mu \mathrm{m})$ is associated with the oldest AFT age (Sample 9, $386 \pm 31 \mathrm{Ma}$ ), while younger ages (ca. $350 \mathrm{Ma}$ to $230 \mathrm{Ma}$ ) are derived from samples displaying shorter MTL $(12.7 \mu \mathrm{m}-11.2 \mu \mathrm{m})$ (Fig. 5). In this case, the fast cooling is associated with a Devonian cooling event and a tail of mixed signatures is showed by samples with AFT ages ranging from Carboniferous to Early Triassic. However, one should be aware of the increased absolute uncertainties on an AFT age when extrapolating further in time and therefore the Devonian basement cooling event is not precisely constrained.
The inverse models also confirm the distinct difference in thermal history between the Araçuaí Orogen, the SFC and the Brasília Orogen (Fig. 6). Samples from the latter two display similar time-temperature paths with a fairly rapid cooling between ca. 430 Ma to 350 Ma with a subsequent phase of stability until a last cooling pulse during the Cenozoic. Almost all the modeled samples from Brasília Orogen and the SFC had cooled below $70^{\circ} \mathrm{C}$ before $250 \mathrm{Ma}$, and were probably not reheated above this temperature since. In the Araçuaí Orogen, the current surface rocks seem to have cooled through the $100{ }^{\circ} \mathrm{C}$ isotherm during the late Mesozoic to the early Cenozoic times, and so therefore the former thermal history is not revealed in the AFT data. The models of samples from the central and southern transect reveal a cooling phase from 150 to $95 \mathrm{Ma}$, i.e. Early Cretaceous to Cenomanian, to recent that indeed is in accordance with their AFT age vs. MTL plot (Fig. 5). Almost all samples describe a modeled late cooling phase that affected all the provinces. In some cases, this cooling phase is inferred during the Late Cretaceous to Paleocene and in others during the Neogene. However, as will be discussed in the next section, this cooling phase is often outside the AFT detection limit (below $60{ }^{\circ} \mathrm{C}$ ) and needs to be confronted with available geological evidence.

\subsection{Meaning of the cooling phases}

\subsubsection{Paleozoic}

A Paleozoic cooling phase is revealed through the thermochronometry and thermal history modeling of samples from the Brasília Orogen and the SFC. The main cooling was constrained between ca. $430 \mathrm{Ma}$ and $350 \mathrm{Ma}$ (Fig. 6) by the inverse modeling (expected model) and corroborated by the relatively high mean track length (MTL) values from sample 9 with a Devonian AFT age. The cooling phase is however poorly constrained since limited suitable basement outcrops and samples are available and only just one sample (sample 9) yields the long MTL signal. In addition, the absolute uncertainty increases for older ages. Previous AFT data have also revealed a Paleozoic cooling period in the southern Brasilia Orogen (Doranti-Tiritan et al., 2014; Fonseca et al., 2020; Gallagher et al., 1994; Ribeiro et al., 2005) and in the north-eastern SFC (Jelinek et al., 2014; Japsen et al., 2012). Fonseca et al., 2020 suggest a close connection between the tectonic subsidence of the Paraná Basin and the coeval cooling of the Brasília Orogen. This is interpreted as a result of exhumation after possible reactivation and basement denudation, which in turn was triggered by the tectonic extension during the rifting processes.

In the interior of West Gondwana, the Paleozoic marks the final consolidation of the supercontinent and the collapse of the Brasiliano orogens. The latter was accompanied by the formation of several intracratonic depocenters. The collapse of the Brasília Orogen specifically is constrained by medium-temperature thermochronometers as to have occurred between $600 \mathrm{Ma}$ to $560 \mathrm{Ma}$ (Hasui and Almeida, 1970; Valeriano et al., 2000; Pimentel et al., 2004; Hackspacher et al., 2004). Hence this implies termination of the main orogenic activity followed by the relaxation of the upwardly compressed isotherms. Dunlap (2000) estimates an initial cooling rate driven by isothermal relaxation in excess of $30^{\circ} \mathrm{C} / \mathrm{Ma}$ using thermomechanical models. Therefore, this initial cooling effect only lasts for about 10 to $20 \mathrm{Ma}$ and thus does not fully explain the reported cooling phase as constrained by our AFT data.

Furthermore, the Brasília Orogen is not the only domain exhibiting this Paleozoic cooling. As mentioned, this signal extends into the SFC and is spread across sampling profiles as far apart as $800 \mathrm{~km}$ and hence implies large, regional mechanisms. We suggest that the interplay between intraplate tectonic readjustments followed by erosional processes led to the basement cooling, i.e. Brasília Orogen and SFC. During the Paleozoic both sampled areas were paleohighs that were subjected to erosion, which produced sediments transported to and deposited in the adjacent depocenters of the Paraná and Sanfranciscana basins. Provenance studies of the Paleozoic sediments of the Paraná 
Basin using paleocurrent data (Alessandretti et al., 2016; Assine, 1999; Assine et al., 1998; Gesicki et al., 2002; Lobato and Borghi, 2005; Mottin et al., 2018; Scherer and Lavina, 2006) and U-Pb detrital zircon analysis (Canile et al., 2016) indeed points to these exhumed northern Precambrian batholiths as possible source areas. Therefore, we link the rapid basement cooling to the significant increase in erosion rates during this period (i.e. Silurian to early Carboniferous). The erosional response of an elevated area (such as the Brasília Orogen and the SFC during the Paleozoic) depends on an intricate set of variables, such as the subsidence of intra-cratonic depocenters, the local climate, the nature of the main erosional process (e.g. fluvial, glacial, arid processes), and base level drop (Braun and Robert, 2005; Dunlap, 2000). The possible individual influences of these processes and their interaction are discussed in Section 5.3.

\subsubsection{Early Cretaceous to Cenomanian}

An Early Cretaceous to Cenomanian (ca. 150 to $95 \mathrm{Ma}$ ) cooling phase is inferred by the inverse models from samples in the southern and central transect from the Araçuaí Orogen. Jelinek et al. (2014) and Carmo (2005) also reported this phase in an adjacent area (Fig. 1). The authors relate it with a denudational event triggered by the surface uplift of the rift shoulder, as the Atlantic opening occurred at this time. The role of the erosion is supported by the offshore syn-rift deposits in the offshore basins next to the Araçuaí Orogen. The influence of the break-up is clearly relevant for the change of the base levels and drainage patterns (e.g. Gallagher et al., 1994; Van Ranst et al., 2020a). The effect of the heat flow within the developing rift system cannot be ruled out (e.g. Brown et al., 1990; Gallagher et al., 1994; Morais Neto et al., 2009; Turner et al., 2008). The effects of the thermal influence decrease rapidly with the distance from the rift axes (Morgan, 1983; Gallagher et al., 1994; Cogné et al., 2011) and as our samples are from at least $150 \mathrm{~km}$ from the coast (Fig. A.1 in Appendix B), its effect might have been minimal. Therefore, we support the Jelinek et al. (2014) hypothesis of a riftshoulder-related denudation as the main cause of cooling during the syn-rift phase.

\subsubsection{Late Cretaceous/Paleocene and Neogene}

Our thermal history models display a possible post-rift rapid cooling phase during the Late Cretaceous to Paleocene (samples S13, C2, N3, N5, N11, Fig. A.8-9 in Appendix B) or during the Neogene (samples N10, C18, C14, C16, Fig. A.9-10 in Appendix B). This final cooling carried the samples from higher temperature conditions to below $65{ }^{\circ} \mathrm{C}$, which is very close to the method's sensitivity. A "recent-cooling" artefact is well-known in numerical inversion of AFT data (e.g. Jonckheere, 2003) and this feature in the models should be rigorously tested against independent geological evidence.

Previous thermochronological studies, in both the Araçuaí Orogen (Amaral-Santos et al., 2019; Carmo, 2005; Jelinek et al., 2014; Van Ranst et al., 2020a) and the SFC (Japsen et al., 2012; Jelinek et al., 2014), also revealed a Late Cretaceous to Paleocene cooling. Many samples from published data present AFT central ages ranging from ca. $80 \mathrm{Ma}$ to $50 \mathrm{Ma}$ with high MTL (> $13.5 \mu \mathrm{m}$ ) and showed narrow standard deviation for the track-length distribution, which means a fairly fast basement cooling between ca. 80 to $50 \mathrm{Ma}$. Thus, we consider that this cooling period may have taken place in some samples of our study area as well. The contemporaneous increase of sedimentary thickness recorded in the offshore basins (Milani et al., 2007), also corroborates increased sedimentary input and more widespread erosion during the Late Cretaceous to Paleocene (e.g. Jelinek et al., 2014).

A Neogene cooling phase is discussed by some studies on the Brazilian margin (Japsen et al., 2012; Jelinek et al., 2014; Morais Neto et al., 2009), however it is solely inferred from thermal history models, since none of the samples showed Neogene AFT central ages. The authors highlight that a thick clastic wedge, built out in the offshore basins during this time, is evidence of some erosion and points to significant basement denudation in the source areas. In our data, the Neogene event is displayed by samples far from the coast, where only thin and small sedimentary deposits are recorded. Even though the inferred cooling may represent the most distal effects of enhanced erosion along the Atlantic margin affecting some samples (e.g. N3 and N5), we support the idea that for the most of the samples this cooling is most likely a modeling artefact than an actual event (e.g. Van Ranst et al., 2020a; Dempster and Persano, 2006; Redfield, 2010).

\subsection{Driving forces}

\subsubsection{Paleozoic}

As discussed, a Paleozoic denudational cooling phase (Fig. 6) affected at least the SFC (Jelinek et al., 2014; Japsen et al., 2012 at the craton's continental margin) and Brasília Orogen (Fonseca et al., 2020). In the Matiqueira Province (Borba et al., 2002, 2003; Hueck et al., 2019; Machado et al., 2019; Oliveira et al., 2016; Ribeiro et al., 2005; Souza et al., 2014), Amazonian Craton (Harman et al., 1998) and ancient West Gondwana basement terrains in Africa (Kasanzu, 2017; Kasanzu et al., 2016), a Paleozoic cooling is also reported. The majority of the previous works argued for a far-field mechanism inducing widespread erosion and exhumation of the West Gondwana basement during this time (e.g. Fonseca et al., 2020). It is likely that other portions of the West Gondwana basement have also experienced this cooling phase but it is not recorded by low-temperature thermochronometers due to either posterior resetting by subsequent thermal events, or because more recent exhumation events have removed the overlying basement with this signal, exposing deeper sections of the crust. This may be the case in the Araçuai Orogen, where the former cooling is not preserved for AFT detection. This hypothesis should be tested by applying other medium-T thermochronometers such as Zircon ( $U$ $-\mathrm{Th}) / \mathrm{He}$, Zircon Fission Track or apatite U-Pb dating. Among the processes supporting an increase of erosion rates, depocenter subsidence was widespread in West Gondwana during the Paleozoic, since several intracratonic basins (also called "interior cratonic basins" or "intracontinental sags") were generated during this time (Torsvik and Cocks, 2013), invoking base level drop and denudation of adjoining basement highs. For example, near the SFC and Brasília Orogen, the extensive Paraná Basin (Milani and De Wit, 2008; Linol et al., 2015; Zalán et al., 1990) shows high rates of episodic tectonic subsidence between the Ordovician (ca. $450 \mathrm{Ma}$ ) and the Early Triassic (ca. $240 \mathrm{Ma}$ ), which is coeval with the Paleozoic cooling period. As previously mentioned, (Section 5.2), provenance studies on Paraná Basin sediments reveal that basement blocks of the SFC and the Brasília Orogen served as important source areas at that time (Alessandretti et al., 2016; Assine, 1999; Assine et al., 1998; Gesicki et al., 2002; Lobato and Borghi, 2005; Mottin et al., 2018; Scherer and Lavina, 2006). Therefore, the Paleozoic cooling of the analyzed basement rocks is likely associated with the basement erosion and sediment influx to the Paraná basin. Three thick sedimentary packages with a total thickness of $4500 \mathrm{~m}$ were deposited in the Paraná basin during this time (Milani et al., 2007). In addition, Paleozoic sedimentary sequences are deposited in the Sanfranciscana Basin in the SFC interior as well (Fig. 6).

Although defining the subsidence mechanism of intracontinental sags remains challenging, Milani and Ramos (1998) showed how the subsidence cycles of the Paraná Basin during the Paleozoic may be connected to the Gondwana geodynamic cycles; mainly to the Famatinian and Gondwanides orogeny (Fig. 6) (Pankhurst et al., 2006). Later, this was extended to the Congo and Cape-Karoo basins due to their similarities in their tectonic subsidence curve and stratigraphic correlation with the Paraná Basin (Milani and De Wit, 2008; Linol et al., 2015). Intraplate subsidence and Phanerozoic basement cooling of Precambrian stable areas (i.e. cratons and ancient orogenic belts) have been indeed linked to the effect of far-field tectonic stress variations (e.g. Kohn and Gleadow, 2019; Pinet, 2018).

The glacial nature of the erosional processes during the Ordovician (e.g. Le Heron, 2018; Torsvik and Cocks, 2013) and Carboniferous in 
West Gondwana may have contributed to increased denudation rates. According to Hallet et al. (1996), glacial erosion can increase the overall erosion rate by one or two orders of magnitude compared to fluvial processes. A Carboniferous ice cap probably affected our study area, since it is close to the glacial deposits of the Sanfranciscana Basin and Paraná Basin and paleocurrent data indeed point to a northern source area (Eyles et al., 1993; Mottin et al., 2018). However, evidence of Ordovician ice caps is rare in Brazil, even though some authors describe glacial deposits in nearby basins, i.e. Parnaíba Basin (e.g. Caputo and dos Santos, 2019).

The transition to the Mesozoic was accompanied by increasing arid conditions and a severe decrease in the tectonic subsidence rate in the Paraná Basin, coincident with the end of the orogenic cycle on the SW portion of Gondwana (Fig. 6). The arid environment with shallow base level prevents further deep erosion and leads to very low denudation rates (Bishop, 2007).

\subsubsection{Early Cretaceous to Cenomanian}

The denudation inferred in the syn-rift stage must be linked to the stretching and thinning processes in response to the extensional stress field that resulted in the opening of South Atlantic Ocean and subsequent formation of new oceanic basins. Models using kinematic constrains suggest that tectonic processes (e.g. flexure of the continental lithosphere followed by surface uplift) are the major driving forces on the early evolution of passive margins (Braun and Beaumont, 1989; Weissel and Karner, 1989; Watts, 2012). The surface uplift of the rift shoulders leads to drainage rearrangements and contribute to increase of the sediment influx in the offshore basins (Watts, 2012). Thus, the destructive nature of erosion acting in the new structural architecture of the rifted continental margin generated the exhumation through denudational processes. According to Rouby et al. (2013) the flexural rift-shoulder is eroded away within 10 to $20 \mathrm{Myr}$, which is in agreement with our inferred cooling phase.

The driving mechanism that led to break-up remains controversial. A mantle plume is commonly invoked to explain the South Atlantic continental rupture (e.g. Quirk et al., 2013 and references therein), mainly because it was followed by huge magmatic events (e.g. ParanáEtendeka L.I $\cdot$ P, with a volume of at least $1,700,000 \mathrm{~km}^{3}$; Frank et al., 2009). However, the original model proposed by Morgan (1971) predicted several phenomena (Campbell, 2007) that the South Atlantic margin fails to show (e.g. Foulger, 2018; Peace et al., 2019; Fromm et al., 2017). Our thermochronological data are insufficient to broadly contribute to this discussion, but brings more insights into the denudation history of the continent's interior. Nevertheless, there is no evidence for regional surface uplift followed by denudation in the Brasília Orogen preceding the emplacement of the Paraná L.I·P that occurred nearby our study area. A kilometer-scale domal surface uplift is predicted in response to plume-head emplacement at the base of the lithosphere (Campbell, 2007). Nor do we observe reheating effects of the Early Cretaceous volcanic activity in the SFC, i.e. Transminas mafic dike swarm, in our data.

\subsubsection{Late Cretaceous - Paleocene}

Our data combined with previous studies (e.g. Amaral-Santos et al., 2019; Cogné et al., 2011; Hueck et al., 2019; Japsen et al., 2012; Jelinek et al., 2014; Krob et al., 2019; Oliveira et al., 2016; Machado et al., 2019; Van Ranst et al., 2020a) show a widespread erosional denudation during the Late Cretaceous to Paleocene that is strongly imprinted in some of the segments of the Brazilian Atlantic margin, such as the Borborema Province (e.g. Morais Neto et al., 2009) and Mantiqueira Province (e.g. Van Ranst et al., 2020a), but is also inferred in thermal history models for samples far away from the margin (e.g. Amaral-Santos et al., 2019; our results) and in the cratonic coastal region (e.g. Japsen et al., 2012). Thus, the Late Cretaceous to Paleocene cooling phase is widespread in the passive marginal domains as well as in the hinterland, which leads us to suggest that a post-rift stress source with only local effects, could not be the main root cause for the basement cooling.

Numerical modeling by Rouby et al. (2013) demonstrated that post-rift peaks of erosion and sediment accumulation should not be explained only by thermal relaxation and flexural isostasy. A major climate change would be one potential explanation for increasing erosion rates, nonetheless, the currently available data point to stable climate conditions throughout that period (Bershaw et al., 2010; Garcia et al., 2005; Spier et al., 2006). A forced tectonic uplift along pre-existing faults and variations in drainage organization seems most likely in our case, as already stated by other authors (e.g. Van Ranst et al., 2020a; Jelinek et al., 2014). Indeed, in SE Brazil, vertical motions along inherited Precambrian structures (crustal weak zones) are well documented (e.g. Cogné et al., 2012). However, the surface uplift/subsidence mechanisms that could trigger such rejuvenation of the topography remain poorly understood.

Paleostress analyses (Salomon et al., 2015; Ferrari, 2001) indicate a compressional NE-SW stress field operating in SE Brazil during the Late Cretaceous to Paleocene in a strike-slip regime. The stress system may be a result of the interplay between flexural bending of the margin and the intraplate compressive stress transmitted from the plate boundaries. Even though the former might not be the only cause of the surface uplift (Salomon et al., 2015), it may attribute to it (Van Ranst et al., 2020a). The Atlantic spreading rate seems to have increased somewhat ( 4 to $5 \mathrm{~cm} / \mathrm{a}$ ) between 90 and $80 \mathrm{Ma}$ to then decrease ( 5 to $3 \mathrm{~cm} / \mathrm{a}$ ) until $60 \mathrm{Ma}$ (Clark, 2018). Thus, a ridge push force cannot be considered as the main cause of stress. At the convergent western boundary of the South American plate, the Peruvian tectono-orogenic cycle took place just before the denudational exhumation we observe (Fig. 6) and so could lead to the increase the intraplate stress, which could in turn trigger the reactivation of ancient structures resulting in differential surface uplift and denudation of certain fault blocks. This hypothesis is commonly proposed to explain the compressional events and cooling periods of the Brazilian passive margin (e.g. Cobbold et al., 2001; Cogné et al., 2011, 2012; Karl et al., 2013; Van Ranst et al., 2020a) and our data can extend this interpretation to terrains more in the hinterland.

\subsection{The influence of tectonic and lithospheric inheritance}

The differential exhumation pattern revealed in our AFT analyses allows clustering the samples in two distinct main groups: (1) samples from the SFC and the Brasília Orogen that were exhumed mainly during the Paleozoic with slight exhumation in the Cenozoic; (2) Samples from the Araçuaí Orogen that were mainly exhumed during the MesoCenozoic.

A first discriminating characteristic is the distance to the coast. The Araçuaí Orogen is part of the Brazilian passive margin and thus closest to the rift axis and would have been more susceptible to heating and denudation processes caused by the rifting (e.g. rift shoulder uplift/ flexural isostasy). However, in the AFT central age vs. distance to coast plot (Fig. A.1 in Appendix B), it is possible to see that between 200 and $300 \mathrm{~km}$ away from the margin, there are several samples from both groups maintaining the characteristics that differentiate them (i.e. older ages in the SFC and Brasillia Orogen). Besides, in the light of previous thermochronological data, the cratonic margin (Japsen et al., 2012; Jelinek et al., 2014; Harman et al., 1998) exhibits much more pre-rift AFT central ages than the orogenic margin (Fig. 7.A), which seldomly preserves the former cooling but rather displays syn- and post-rift AFT ages. Therefore, we suggest that lithospheric features may be decisive in the manifestation of surface uplift and the denudation and cooling afterwards, at least during the Meso-Cenozoic events.

The Meso-Cenozoic tectonic activity should be mainly partitioned in pre-existing shear zones within the lithosphere, as shown by the parallelism between ancient structural lineaments of the Brasiliano belts and the younger rift systems, e.g. Atlantic Rift and Continental Rift of 


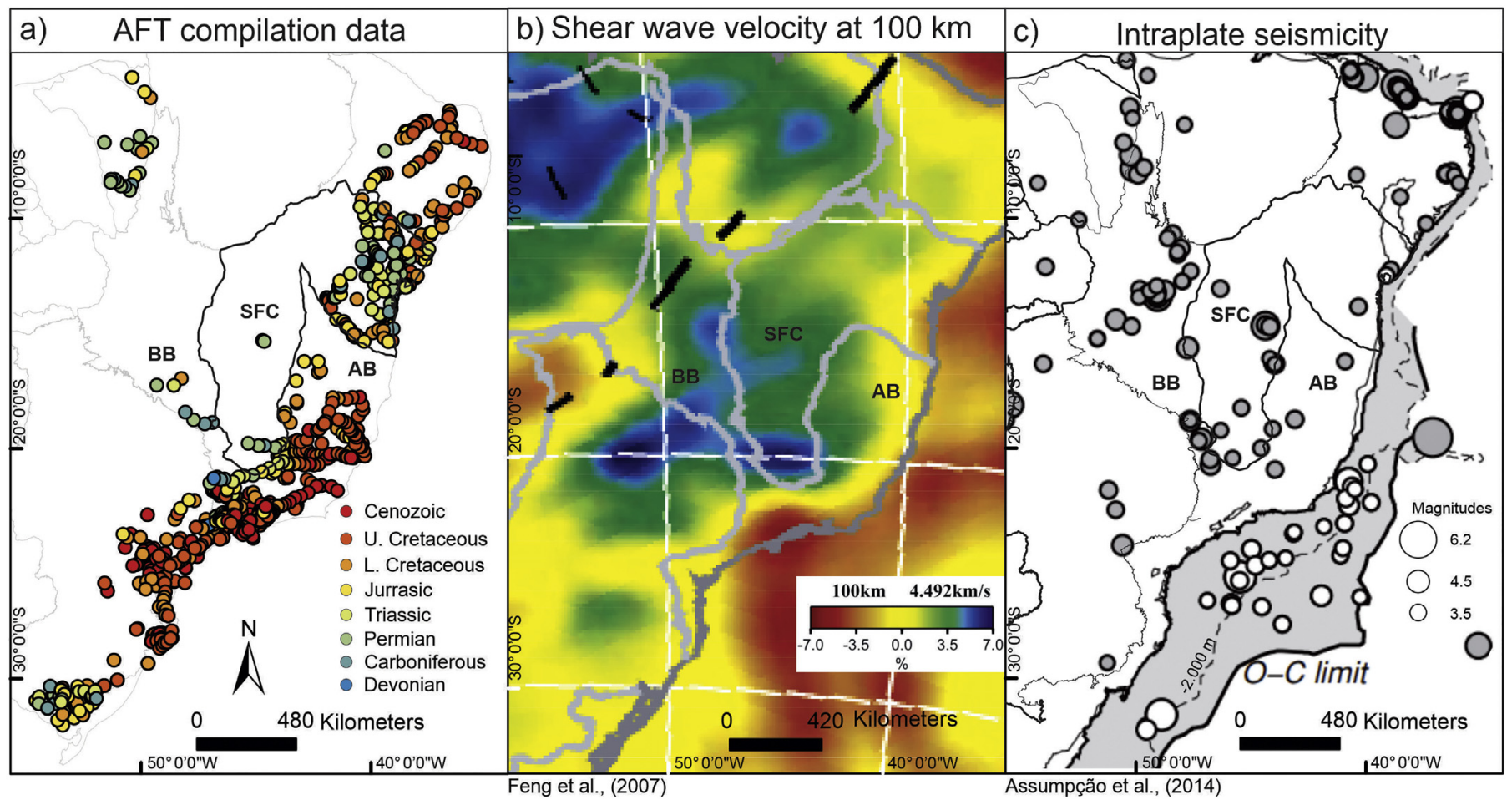

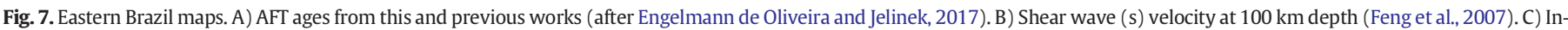
traplate seismicity onshore and offshore (Assumpção et al., 2014).

south-eastern Brazil (Ashby et al., 2010; Tommasi and Vauchez, 2001, 2015; Reuber and Mann, 2019; Salomon et al., 2017; de Wit et al., 2008; Nemčok, 2016). The mobile belts indeed possess several weak zones, such as faults, shear zones, and foliation planes, as well as a higher geothermal gradient (Misra and Mukherjee, 2015; Cloetingh et al., 1995; Rey et al., 2001; Corti et al., 2007). These features generate a warmer and weaker lithosphere compared to the cratonic one. Cratons are characterized by low heat flux, and a dehydrated mantle with refractory composition (e.g. Peslier et al., 2010; Sleep, 2003). Tommasi and Vauchez $(2001,2015)$, taking into account these characteristics, suggest that the orogenic mantle provides deep pre-existing weak zones as well, which play an important role in localizing strain during extension such as in the opening of the South Atlantic Ocean.

Hence, the South Atlantic margin gives a good example of how cratonic and orogenic lithospheres can create different features undergoing the same geodynamic event (e.g. Brune et al., 2014; Buck, 1991; Huismans and Beaumont, 2005). The break-up of the Bahia-Gabon cratonic bridge between the SFC and the Congo Craton resulted in deep narrow basins (marginal basins and the Recôncavo-Tucano-Jatobá aulacogen), while portions of extended orogenic belts (e.g. Araçuaí Orogen) generated a wider rifting zone (e.g. Mohriak et al., 2008). We earlier suggested the erosion of the rift shoulders as the main cause of the Early Cretaceous to Cenomanian cooling phase (Section 5.3). It is absent in our in-land cratonic study area and also in some cratonic coastal portions (Jelinek et al., 2014) thus the deformation, i.e. flexural surface uplift, should not have regionally reached the cratonic strong lithosphere. Instead, and more likely, the deformation in the SFC was heterogeneously localized in narrow weak zones (e.g. Recôncavo-TucanoJatobá rift) and at the cratonic edges. In contrast, the mobile marginal belts (e.g. Araçuaí, Borborema and Ribeira belts) exhibit widespread Meso-Cenozoic denudational phases indicating far-reaching deformation. Distinct erosion patterns of cratonic rocks versus rocks from the boundary orogenic belts, are reported from the southern Africa Plateau as well, and is thought to stem from the same geodynamic driving force (Stanley et al., 2015).
Although the dichotomy between cratonic vs. orogenic lithosphere works to understand the general context, it is too simplistic if we consider the several heterogeneities within one lithosphere or between two cases of the same lithospheric type. Fig. 7.B is a tomographic image that displays the S-wave velocity model at $100 \mathrm{~km}$ depth (Feng et al., 2007). The higher S-wave velocity terrains are indeed underlain by old and stable cratonic lithosphere, however the tomography model shows that the thickness of the lithosphere is not homogeneous in the SFC and it is higher beneath its southern part (Feng et al., 2007). Surrounding the SFC, the Brasília and Araçuaí orogens present very different lithospheric architectures, which is also illustrated in Fig. 7.B. The Brasília Orogen in our study area exhibits significantly faster S-wave velocities than the Araçuaí Orogen, which is interpreted as a deeperseated extension of the SFC beneath the former orogen (Soares et al., 2006; Feng et al., 2007). In order to better understand the individuality of each region it is necessary to take into account the formation and evolution of the lithosphere over time.

In our SFC data, samples nearby the cratonic boundary (samples C1, $\mathrm{C} 22, \mathrm{~S} 11, \mathrm{~S} 13, \mathrm{~S} 14)$ present the youngest AFT central ages, ranging from Triassic to Jurassic, along with MTL values that are higher than $12.5 \mu \mathrm{m}$. Comparing to the intraplate seismicity analyses of Brazilian earthquakes (Fig. 7.C) (compilation of Brazilian Seismic Bulletin and previous works), it is possible to note that the seismicity is more concentrated at the cratonic edges as compared to the inner craton (Assumpção et al., 2013), which is in agreement with observations by Mooney et al. (2012) on a global scale. The latter authors suggest that the increase in the seismic activity can be a consequence of stress concentration in areas with high lateral density variations. The stress concentration might result in more extensive reactivation of the basement, leading to faster exhumation, shorter APAZ residence time and thus the long MTL values observed for samples in the cratonic boundary as compared to the length distributions of the samples of the inner craton.

In the case of an orogenic lithosphere, factors that facilitate reworking or reactivation are well-understood, however many orogenic belts do not show evidence of later tectonic activity in extensive 
large-scale (Krabbendam, 2001). This could indeed be the case for the Brasília Orogen, since our data show little post-Paleozoic reactivation for samples from that domain. Krabbendam (2001) categorized the Brasília Orogen as "unlikely to be reworked", based on analyses of its stronger orogenic lithosphere. We suggest that the Brasília Orogen is a case of strong orogenic lithosphere capable to inhibit reactivation, at least compared to the weaker Araçuaí Orogen lithosphere. Processes that could lead to the relatively high lithospheric strength include the enrichment of radioactive isotopes in the shallow crust with depletion of the lower crust, dehydration metamorphism, and crustal thinning by erosion (Krabbendam, 2001). These processes are responsible to lower the geothermal gradient and consolidate a higher strength rheology to deeper-seated rocks.

The Brasília Orogen was formed by the closure of the wide Goiás ocean (Brito Neves, 1999; Pimentel, 2016) resulting in the incorporation of high amounts of mafic material and an island arc terrain (Valeriano, 2017 and references therein), which are depleted in radiogenic elements. In addition, in our study area, there are many granulitic rocks including mafic granulites (e.g. Moraes et al., 2002; Piuzana, 2003) that indicate emplacement temperatures exceeding $1000{ }^{\circ} \mathrm{C}$ (Del Lama et al., 2000), and have resulted in a high strength dehydrated mineral assemblage. During the metamorphism, the partial melting also contributed to the depletion of incompatible radioactive elements in the lower crust. Finally, the orogenic collapse does not seem to have an evolved extensional phase, neither abundant magmatism (Carvalho et al., 2014), thus the erosion of the upper crust may have been the main process of crustal thinning. The removal of the upper crust enriched in radiogenic elements produced an orogen depleted in radiogenic heat and thereby led to a colder and stronger terrain. Another important feature revealed by the seismic tomography (Feng et al., 2007) that points to an increased lithospheric stability is the above mentioned deep subcontinental lithospheric mantle in the south of the orogen.

In the Araçuaí Orogen, the samples from the northern transect yield AFT central ages ranging from the Late Cretaceous to the Jurassic and MTL values $(11.5 \mu \mathrm{m})$ lower than in the central and southern transect $(\mathrm{MTL}>12.2 \mu \mathrm{m})$. The thermal history models from samples N3 and N5 (Fig. 6) reflect these data, showing a slow cooling since the Jurassic, in contrast to the other models from the Araçuaí Orogen that rather infer a fairly fast cooling just after the Early Cretaceous. This could be an effect of the distance to the coast or elevation (see Fig. A.1 and A.2 in Appendix B), since these samples follow the normal trends defined by the dataset from this orogen. However, the crustal level exposed in the northern segment of the Araçuai Orogen (north of $19^{\circ} \mathrm{S}$ ) is notably shallower than in the south (Alkmim et al., 2017; Pedrosa-Soares et al., 2011), which would mean that this sector was in general less deeply eroded, i.e. went through a slower denudational cooling. The evidence of the exposure of different crustal levels in the north of the orogen include the decrease in the metamorphic grade and the occurrence of shallower portions of the post-orogenic batholiths, i.e. much less mafic components (Pedrosa-Soares et al., 1992, 2001, 2011; Trompette, 1994; Pedrosa-Soares and Wiedemann-Leonardos, 2000).

A widespread sampling for thermochronological analysis in the northern sector of Araçuaí Orogen would cover samples closest to the margin and in a variety of elevations, therefore the influence of these variables could be checked. If a broad protracted cooling were to be revealed such as in our data, we would suggest that the degree of erosion contributes to the preservation of the upper crust in the northern sector. In this case the reactivations responsible to induce the denudational processes to the south would produce slower vertical motion to the north. The rigidity of the northern lithosphere is increased by the proximity to the more rigid cratonic bridge and a lesser amount of extension during the formation of precursor basin of the orogen. The analysis of the structural trend is also relevant, since it inflects to the NE in the north portion making it less parallel to the coastal margin and consequently less favorable to react to extensional forces inflicted by opening of the South Atlantic (e.g. Salomon et al., 2017). Ideally, the contribution of the lithospheric and structural inheritance in this segment of the belt should be clarified in future works.

\section{Conclusions}

We report the results of AFT thermochronology from forty-three new samples distributed in three transects that run across the São Francisco Craton (SFC) and two adjacent mobile orogenic belts, i.e. the Araçuaí and Brasília belts. Our analyses in combination with published data reveal three main phases of denudation during the Phanerozoic: (i) Paleozoic, recorded by samples from the SFC and Brasília Orogen; (ii) Early Cretaceous to Cenomanian, recorded by samples from the Araçuaí Orogen, and (iii) Late Cretaceous to Paleocene, inferred in samples from the SFC as well as from both the Brasília and Araçuaí orogens. We associated the Paleozoic phase with erosion induced by the effect of tectonic extension during the rifting of continental crust in the context of tectonic subsidence of the intracontinental basins, i.e. mainly the Paraná and Sanfranciscana basins. The Early Cretaceous to Cenomanian cooling was linked with the uplift induced denudation triggered by the opening of the South Atlantic Ocean during the syn-rift phase. Finally, the widespread post-rift erosional event, i.e. Late Cretaceous to Paleocene, was connected with reactivations prompted by the interplay between flexural bending of the margin and the intraplate compressive stress transmitted from the plate boundaries.

Differential uplift and exhumation in our study area are recorded in our AFT data. While the Araçuaí Orogen samples experienced fairly rapid cooling during Meso-Cenozoic times, the SFC and Brasília Orogen samples exhumed long before, more specifically during the Paleozoic, and remained in relative stable conditions near present outcrop position from the Mesozoic until the present day. We suggest that the lithospheric architecture of the tectonic provinces is deeply connected with deformational response to tectonic stresses. The strong, rigid and cold cratonic lithosphere likely partitioned and concentrated the Meso-Cenozoic reactivations in narrow weak zones (e.g. Recôncavo-Tucano-Jatobá rift) and at the cratonic borders as evidenced also by previous thermochronological studies in the cratonic basement of the Brazilian margin (Jelinek et al., 2014). In contrast, the Araçuaí Orogen displays a widespread Meso-Cenozoic denudational exhumation indicating farreaching deformation. The Brasília Orogen seems to be an example of a strong orogenic lithosphere, since it remained stable from the early Mesozoic to the present. Therefore, we highlight the importance of inherited lithospheric structures in studies elucidating thermal histories.

\section{Declaration of Competing Interest}

The authors declare that they have no known competing financial interests or personal relationships that could have appeared to influence the work reported in this paper.

\section{Acknowledgments}

This work was supported by the Conselho Nacional de Desenvolvimento Científico e Tecnológico - CNPq (Project 306520/2018-4). The first authors thank Coordenação de Aperfeiçoamento de Pessoal de Nivel Superior - CAPES (the research fund of the Brazilian Ministry of Education) for the master's scholarships. TFB acknowledges the Fundação de Amparo Pesquisa do Estado de Minas Gerais (FAPEMIG) for the Ph.D scholarship funding. SN's contribution was supported by the Research Foundation - Flanders (FWO) through a PhD fellowship 31528111. GVR is supported by the Special Research Fund of Ghent University (BOF 01N03915) We are grateful to Dr. Bart Van Houdt for performing the irradiations at the SCK in Mol (Belgium) and Fernando Pacheco for helping to improve the figures. 


\section{Appendix A. Supplementary data}

Supplementary data to this article can be found online at https://doi. org/10.1016/j.gr.2021.01.006.

\section{References}

Alessandretti, L., Machado, R., Warren, L.V., Assine, M.L., Lana, C., 2016. From source-tosink: the Late Permian SW Gondwana paleogeography and sedimentary dispersion unraveled by a multi-proxy analysis. J. South Am. Earth Sci. 70, 368-382. https:// doi.org/10.1016/j.jsames.2016.06.007.

Alkmim, F.F., 2004. O que faz de um cráton um cráton? O cratón São Francisco e as revelações almeidianas ao delimitá-lo. In: Mantesso-Neto, V., Bartorelli, A., Carneiro, C.D.R., Brito Neves, B.B. (Eds.), Geologia do Continente Sul-Americano: Evolução Da Obra de Fernando Flávio Marques de Almeida. Editora Beca, São Paulo, pp. 17-35.

Alkmim, F.F., 2015. Geological background: a tectonic panorama of Brazil. In: Vieira, B.C., Salgado, A.A.R., Santos, L.J.C. (Eds.), Landscapes and Landforms of Brazil. Springer, pp. 9-17 https://doi.org/10.1007/978-94-017-8023-0_2.

Alkmim, F.F., Teixeira, W., 2017. The Paleoproterozoic Mineiro Belt and the Quadrilátero Ferrífero. In: Heilbron, M., Cordani, U.G., Alkmim, F.F. (Eds.), São Francisco Craton. Springer, Eastern Brazil, pp. 71-94 https://doi.org/10.1007/978-3-319-01715-0.

Alkmim, F.F., Brito Neves, B.B., Alves, J.A.C., 1993. Arcabouço tectônico do Cráton do São Francisco - uma revisão. In: Dominguez, M.L., Misi, A. (Eds.), O Cratón Do São Francisco: Trabalhos Apresentados Na Reunião Preparatória Do II Simposio Sobre o Cráton Do São Francisco. SBG-SGM-CNPq, Salvador, pp. 45-62.

Alkmim, F.F., Kuchenbecker, M., Reis, H.L.S., Pedrosa-Soares, A.C., 2017. The Araçuaí Belt. In: Heilbron, M., Cordani, U.G., Alkmim, F.F. (Eds.), São Francisco Craton, Eastern Brazil. Springer, pp. 255-275 https://doi.org/10.1007/978-3-319-01715-0.

Almeida, F.F.M., 1977. O Craton do Säo Francisco. Rev. Barsil. Geoci. 7, 349-364. https:// doi.org/10.5327/rbg.v7i4.128.

Almeida, F.F.M., De Brito Neves, B.B., Dal Ré Carneiro, C., 2000. The origin and evolution of the South American Platform. Earth Sci. Rev. 50, 77-111. https://doi.org/10.1016/ S0012-8252(99)00072-0.

Almeida, F.F.M., Hasui, Y., Brito Neves, B.B., Fuck, R.A., 1981. Brazilian structural provinces: an introduction. Earth Sci. Rev. 17, 1-29. https://doi.org/10.1016/0012-8252(81) 90003-9.

Amaral-Santos, E., Jelinek, A.R., Genezine, F.A., 2019. Phanerozoic cooling history of Archean/Paleoproterozoic basement in the southern Espinhaço Range, southeastern Brazil, through apatite fission-track analysis. J. South Am. Earth Sci., 102352 https:// doi.org/10.1016/j.jsames.2019.102352.

Artemieva, I.M., 2006. Global $1^{\circ} \times 1^{\circ}$ thermal model TC1 for the continental lithosphere: implications for lithosphere secular evolution. Tectonophysics 416, 245-277. https:// doi.org/10.1016/j.tecto.2005.11.022.

Ashby, D., Mccaffrey, K., Holdsworth, B., Almeida, J., 2010. Structural controls on the evolution of the Southeastern Brazilian continental margin. Geophysical Research Abstracts. EGU General Assembly.

Asmus, H.E., 1982. Geotectonic significance of Mesozoic-Cenozoic magmatic rocks in the Brazilian continental margin and adjoining emerged area, in: Congresso LatinoAmericano de Geologia. Servicio Geologico Nacional, Buenos Aires, pp. 761-779.

Assine, M.L., 1999. Fácies, icnofósseis, paleocorrentes e sistemas deposicionais da Formação Furnas no flanco sudeste da Bacia do Paraná. Rev. Bras. Geociências 29, 357-370.

Assine, M.L., Perinotto, J.A., Fulfaro, V.J., Petri, S., 1998. Progradação deltaica tibagi no devoniano médio da Bacia do Paraná. Rev. Bras. Geoci. 28, 125-134. https://doi.org/ 10.1023/A:1014771612802.

Assumpção, M., Feng, M., Tassara, A., Julià, J., 2013. Models of crustal thickness for South America from seismic refraction, receiver functions and surface wave tomography. Tectonophysics 609, 82-96. https://doi.org/10.1016/j.tecto.2012.11.014.

Assumpção, M., Ferreira, J., Barros, L., Bezerra, H., França, G.S., Barbosa, J.R., Menezes, E., Carlos Ribotta, L., Pirchiner, M., do Nascimento, A., Dourado, J.C., 2014. Intraplate seismicity in Brazil. In: Talwani, P. (Ed.), Intraplate Earthquakes. Cambridge University Press, Cambridge, pp. 50-71 https://doi.org/10.1017/CBO9781139628921.004.

Autin, J., Bellahsen, N., Leroy, S., Husson, L., Beslier, M.O., d'Acremont, E., 2013. The role of structural inheritance in oblique rifting: Insights from analogue models and application to the Gulf of Aden. Tectonophysics 607, 51-64. https://doi.org/10.1016/j. tecto.2013.05.041

Barbosa, J.S.F., Barbosa, R.G. 2017. The paleoproterozoic Eastern Bahia orogenic domain. In: Heilbron, M., Cordani, U.G., Alkmim, F.F. (Eds.), São Francisco Craton. Springer, Eastern Brazil, pp. 57-69 https://doi.org/10.1007/978-3-319-01715-0.

Batezelli, A. 2017. Continental systems tracts of the Brazilian Cretaceous Bauru Basin and their relationship with the tectonic and climatic evolution of South America. Basin Res. 29, 1-25. https://doi.org/10.1111/bre.12128.

Bershaw, J. Garzione, C.N., Higgins, P., MacFadden, B.J., Anaya, F., Alvarenga, H., 2010. Spatial-temporal changes in Andean plateau climate and elevation from stable isotopes of mammal teeth. Earth Planet. Sci. Lett. 289, 530-538. https://doi.org/ 10.1016/j.epsl.2009.11.047.

Bishop, P., 2007. Long-term landscape evolution: linking tectonics and surface processes. Earth Surf. Process. Landforms 32, 329-365. https://doi.org/10.1002/esp.

Borba, A.W., Vignol-Lelarge, M.L.M., Mizusaki, A.M.P., 2002. Uplift and denudation of the Cacapava do Sul granitoids (southern Brazil) durning Late Paleozoic and Mesozoic: constraints form apatite fission-track data. J. South Am. Earth Sci. 15, 683-692.

Borba, A.W., Lima, E.F., Vignol-Lelarge, M.L.M., Mizusaki, A.M.P., Sparrenberg, I., Barros, C.E., 2003. Significance of Late Paleozoic fission-track ages in volcanic rocks from the Lavras Do Sul region, Southernmost Brazil. Gondwana Res. 6, 79-88. https://doi. org/10.1016/S1342-937X(05)70645-6.

Braun, J., Beaumont, C., 1989. Contrasting styles of lithospheric extension: implications for differences between Basin and Range province and rifted continental margins. In: Tankard, A.J., Balkwill, H.R. (Eds.), Extensional Tectonics and Stratigraphy of the North Atlantic Margins. American Association of Petroleum Geologists, pp. 53-79 https://doi.org/10.1306/M46497 ISBN.

Braun, J., Robert, X., 2005. Constraints on the rate of post-orogenic erosional decay from low-temperature thermochronological data: application to the Dabie Shan, China. Earth Surf. Process. Landforms 30, 1203-1225. https://doi.org/10.1002/esp.1271.

Brito Neves, B.B., 1999. América do Sul: quatro fusões, quatro fissões e o processo acrescionário andino. Rev. Bras. Geoci. 29, 379-392.

Brito Neves, B.B., 2002. Main stages of the development of the sedimentary basins of South America and their relationship with the tectonics of supercontinents. Gondwana Res. 5, 175-196.

Brown, R.W., Rust, D.J., Summerfield, M.A., Gleadow, A.J.W., De Wit, M.C.J., 1990. An early cretaceous phase of accelerated erosion on the south-western margin of Africa: evidence from apatite fission track analysis and the offshore sedimentary record. Nucl. Tracks Radiat. Meas. 17, 339-350. https://doi.org/10.1016/13590189(90)90056-4

Brune, S., Heine, C., Pérez-Gussinyé, M., Sobolev, S.V., 2014. Rift migration explains continental margin asymmetry and crustal hyper-extension. Nat. Commun. 5. https://doi. org/10.1038/ncomms5014.

Buck, W.R., 1991. Modes of continental lithospheric extension. J. Geophys. Res. 96, 20161-20178. https://doi.org/10.1029/91jb01485.

Buso, V.V., Aquino, C.D., Paim, P.S.G., Souza, P.A., Mori, A.L., Fallgatter, C., Milana, J.P., Kneller, B., 2017. Late Palaeozoic glacial cycles and subcycles in western Gondwana: correlation of surface and subsurface data of the Paraná Basin, Brazil. Palaeogeogr. Palaeoclimatol. Palaeoecol. 531. https://doi.org/10.1016/j.palaeo.2017.09.004.

Campbell, I.H., 2007. Testing the plume theory. Chem. Geol. 241, 153-176. https://doi.org/ 10.1016/j.chemgeo.2007.01.024.

Campos, J.E.G., Dardenne, M.A., 1997. Origem e evolução tectônica da Bacia Sanfranciscana. Rev. Barsil. Geoci. 27, 283-294. https://doi.org/10.5327/rbg.v27i3.596.

Canile, F.M., Babinski, M., Rocha-Campos, A.C., 2016. Evolution of the Carboniferous-Early Cretaceous units of Paraná Basin from provenance studies based on U-Pb, Hf and O isotopes from detrital zircons. Gondwana Res. 40, 142-169. https://doi.org/10.1016/ j.gr.2016.08.008.

Caputo, M.V., Crowell, J.C., 1985. Migration of glacial centers across Gondwana during Paleozoic Era. Geol. Soc. Am. Bull. 96, 1020-1036. https://doi.org/10.1130/0016-7606 (1985) $96<1020$

Caputo, M.V., dos Santos, R.O.B., 2019. Stratigraphy and ages of four Early Silurian through Late Devonian, Early and Middle Mississippian glaciation events in the Parnaíba Basin and adjacent areas, NE Brazil. Earth Sci. Rev., 103002 https://doi.org/10.1016/j. earscirev.2019.103002.

Carmo, I.D.O., 2005. Geocronologia do Intemperismo Cenozóico no Sudeste do Brasil. PhD thesis. Universidade Federal do Rio de Janeiro.

Carneiro, Celso Dal Ré, Almeida, Fernando Flávio Marques, Hasui, Yociteru, Zalán, P.V., Teixeira, J.B.G., 2012. In: Hasui, Y., Carneiro, C.D.R., Almeida, F.F.M., Bartorelli, A. (Eds.), Estágios evolutivos do Brasil no Fanerozóico. Geologia do Brasil, Beca, pp. $131-136$

Carvalho, B.B., de Janasi, V.A., Henrique-Pinto, R., 2014. Geochemical and Sr-Nd-Pb isotope constraints on the petrogenesis of the K-rich Pedra Branca Syenite: Implications for the Neoproterozoic post-collisional magmatism in SE Brazil. Lithos 205, 39-59. https://doi.org/10.1016/j.lithos.2014.06.016.

Cavalcante, C., Hollanda, M.H., Vauchez, A., Kawata, M., 2018. How long can the middle crust remain partially molten during orogeny? Geology 46, 839-842. https://doi. org/10.1130/G45126.1.

Chaves, A.D.O., 2013. Enxames De Diques Máficos De Minas Gerais - O Estado Da Arte Geonomos 21, 29-33. http://dx.doi.org/10.18285/geonomos.v21i1.253.

Clark, S.R., 2018. Uncertainty in the breakup, spreading history, and velocity variations of Gondwana. Gondwana Res. 53, 189-196. https://doi.org/10.1016/j.gr.2017.04.029.

Cloetingh, S., van Wees, J.D., van der Beek, P.A., Spadini, G., 1995. Role of pre-rift rheology in kinematics of extensional basin formation: constraints from thermomechanical models of Mediterranean and intracratonic basins. Mar. Pet. Geol. 12, 793-807. https://doi.org/10.1016/0264-8172(95)98848-Y.

Cobbold, P.R., Meisling, K.E., Mount, V.S., 2001. Reactivation of an obliquely rifted margin, Campos and Santos basins, southeastern Brazil. Am. Assoc. Pet. Geol. Bull. 85, 1925-1944. https://doi.org/10.1306/8626d0b3-173b-11d7-8645000102c1865d.

Coelho, R.M., Chaves, A.O., 2017. Diques máficos de Minas Gerais do Cretáceo Inferior: idades Ar-Ar e correlação com a Província Ígnea Paraná-Etendeka. Geociências 4 , 613-622.

Cogné, N., Gallagher, K., Cobbold, P.R., 2011. Post-rift reactivation of the onshore margin of southeast Brazil: Evidence from apatite (U-Th)/He and fission-track data. Earth Planet. Sci. Lett. 309, 118-130. https://doi.org/10.1016/j.epsl.2011.06.025.

Cogné, N., Gallagher, K., Cobbold, P.R., Riccomini, C., Gautheron, C., 2012. Post-breakup tectonics in southeast Brazil from thermochronological data and combined inverseforward thermal history modeling. J. Geophys. Res. B 117, 1-16. https://doi.org/ 10.1029/2012JB009340.

Corti, G., van Wijk, J., Cloetingh, S., Morley, C.K., 2007. Tectonic inheritance and continental rift architecture: numerical and analogue models of the East African Rift system. Tectonics 26, 1-13. https://doi.org/10.1029/2006TC002086.

De Corte, F., Bellemans, F., Van den Haute, P., Ingelbrecht, C., Nicholl, C., 1998. A new U doped glass certified by the European commission for the calibration of fissiontrack dating. In: van den Haute, P., de Corte, F. (Eds.), Advances in Fission-Track Geochronology. Springer, Dordrecht. 
De Grave, J., Glorie, S., Vermaercke, P., Vittiglio, G., Van den Haute, P., 2010. A “ new " irradiation facility for FT applications at the Belgian Nuclear Research Centre: the BR1 reactor. Thermochronology,12th International Conference.

De Pelsmaeker, E., Glorie, S., Buslov, M.M., Zhimulev, F.I., Poujol, M., Korobkin, V.V., Vanhaecke, F., Vetrov, E.V., De Grave, J., 2015. Late-Paleozoic emplacement and Meso-Cenozoic reactivation of the southern Kazakhstan granitoid basement. Tectonophysics 662, 416-433. https://doi.org/10.1016/j.tecto.2015.06.014.

de Wit, M.J., Brito Neves, B.B., Trouw, R.A.J., Pankhurst, R.J., 2008. Pre-Cenozoic correlations across the South Atlantic region: (the ties that bind). In: Pankhurst, R.J., Trouw, R.A.J., Brito Neves, B.B., de Wit, M.J. (Eds.), West Gondwana: Pre-Cenozoic Correlations Across the South Atlantic Region. Geological Society, London, pp. 1-8 https://doi.org/10.1144/SP294.1.

Del Lama, E.A., Zanardo, A., Oliveira, M.A.F., Morales, N., 2000. Exhumation of highpressure granulites of the Guaxupé Complex, southeastern Brazil. Geol. J. 35, 231-249. https://doi.org/10.1002/gj.859.

Dempster, T.J., Persano, C., 2006. Low-temperature thermochronology: resolving geotherm shapes or denudation histories? Geology 34, 73-76. https://doi.org/ $10.1130 /$ G21980.1.

Donelick, R.A., 1991. Crystallographic orientation dependence of mean etchable fission track length in apatite: an empirical model and experimental observations. Am. Mineral. 76, 83-91.

Donelick, R.A., Ketcham, R.A., Carlson, W.D., 1999. Variability of apatite fission-track annealing kinetics: II. Crystallographic orientation effects. Am. Mineral. 84, 1224-1234. https://doi.org/10.2138/am-1999-0902.

Doranti-Tiritan, C., Hackspacher, P.C., Ribeiro, M.C.S., Glasmacher, U.A., De Souza, D.H., 2014. Relief evolution of Poços de Caldas (SP/MG) Region based in thermochronology data and 3D thermokinematic modeling. Rev. Bras. Geomorfol. 15, 291-310. https:// doi.org/10.20502/rbg.v15i2.491.

Dunlap, W.J., 2000. Nature's diffusion experiment: the cooling-rate cooling-age correlation. Geology 28, 139-142.

Engelmann de Oliveira, C.H., Jelinek, A.R., 2017. História termotectônica da margem continental Brasileira a partir de dados de traços de fissão em apatita. Pesqui. em Geociencias. 44, pp. 387-400. https://doi.org/10.22456/1807-9806.83263.

Engelmann de Oliveira, C.H., Jelinek, A.R., Chemale, F., Cupertino, J.A., 2016. Thermotectonic history of the southeastern Brazilian margin: evidence from apatite fission track data of the offshore Santos Basin and continental basement. Tectonophysics 685, 21-34. https://doi.org/10.1016/j.tecto.2016.07.012.

Enkelmann, E., Jonckheere, R., Ratschbacher, L., 2005. Absolute measurements of the uranium concentration in thick samples using fission-track detectors. Nucl. Instr. Methods Phys. Res. Sect. B 229, 489-498. https://doi.org/10.1016/j.nimb.2005.01.003.

Eyles, C.H., Eyles, N., Franca, A.B., 1993. Glaciation and tectonics in an active intracratonic basin: the Late Palaeozoic Itararé Group, Paraná Basin, Brazil. Sedimentology 40, $1-25$.

Feng, M., van der Lee, S., Assumpção, M., 2007. Upper mantle structure of South America from joint inversion of waveforms and fundamental mode group velocities of Rayleigh waves. J. Geophys. Res. Solid Earth 112, 1-16. https://doi.org/10.1029/ 2006JB004449.

Fernie, N., Glorie, S., Jessell, M.W., Collins, A.S., 2018. Thermochronological insights into reactivation of a continental shear zone in response to Equatorial Atlantic rifting (northern Ghana). Sci. Rep. 8, 1-14. https://doi.org/10.1038/s41598-018-34769-x.

Ferrari, A.L., 2001. Evolução Tectônica do Graben da Guanabara. PhD Thesis. Universidade de São Paulo.

Fleisher, R.L., Price, P.B., Walker, R.M., 1975. Nuclear Tracks in Solids: Principles and Applications. University of California Press, Berkeley, Calif.

Fonseca, A.C., Piffer, G.V., Nachtergaele, S., Van Ranst, G., De Grave, J., Novo, T.A., 2020. Devonian to Permian post-orogenic denudation of the Brasília Belt of West Gondwana: insights from apatite fission track thermochronology. J. Geodyn. 137. https://doi.org/ 10.1016/j.jog.2020.101733.

Fossen, H., Cavalcante, G.C., Almeida, R.P., 2017. Hot versus cold orogenic behavior: comparing the Araçuaí-West Congo and the Caledonian Orogens. Tectonics 36 , 2159-2178. https://doi.org/10.1002/2017TC004743.

Foulger, G.R., 2018. Origin of the South Atlantic igneous province. J. Volcanol. Geotherm. Res. 355, 2-20. https://doi.org/10.1016/j.jvolgeores.2017.09.004.

Frank, H.T., Gomes, M.E.B., Formoso, M.L.L., 2009. Review of the areal extent and the volume of the Serra Geral Formation, Paraná Basin, South America. Pesqui. Geoci. 36, 49-57.

Fromm, T., Jokat, W., Ryberg, T., Behrmann, J.H., Haberland, C., Weber, M., 2017. The onset of Walvis Ridge: plume influence at the continental margin. Tectonophysics 716, 90-107. https://doi.org/10.1016/j.tecto.2017.03.011.

Galbraith, R.F., 1981. On statistical models for fission track counts. Math. Geol. 13, 471-478. https://doi.org/10.1007/BF01034498.

Galbraith, R.F., 2005. Statistics for Fission Track Analysis. Chapman and Hall/CRC, New York

Galbraith, R.F., Laslett, G.M., 1993. Statistical models for mixed fission track ages. Nucl. Tracks Radiat. Meas. 21, 459-470. https://doi.org/10.1016/1359-0189(93)90185-C.

Gallagher, K., 2012. Transdimensional inverse thermal history modeling for quantitative thermochronology. J. Geophys. Res. 117, 1-16. https://doi.org/10.1029/2011JB008825.

Gallagher, K., Hawkesworth, C.J., Mantovani, M.S.M., 1994. The denudation history of the onshore continental margin of SE Brazil inferred from apatite fission track data. J. Geophys. Res. 99, 18117-18145. https://doi.org/10.1029/94JB00661.

Gallagher, K., Hawkersworth, C.J., Mantovani, M.S.M., 1995. Denudation, fission track analysis and the long-term evolution of passive margin topograpy: application to the southeastet Brazilian margin. J. South Am. Earth Sci. 8, 65-77 0895-9811(94) 00042-5.
Garcia, A.J.V., Rosa, Á.A.S., Goldberg, K., 2005. Paleoenvironmental and paleoclimatic control on early diagenetic processes and fossil record in Cretaceous continental sandstones of Brazil. J. South Am. Earth Sci. 19, 243-258. https://doi.org/10.1016/j. jsames.2005.01.008.

Gesicki, A.L.D., Riccomini, C., Boggiani, P.C., 2002. Ice flow direction during late Paleozoic glaciation in Western Paraná Basin, Brazil. J. South Am. Earth Sci. 14, 933-939. https://doi.org/10.1016/S0895-9811(01)00076-1.

Gillespie, J., Glorie, S., Xiao, W., Zhang, Z., Collins, A.S., Evans, N., McInnes, B., De Grave, J. 2017. Mesozoic reactivation of the Beishan, southern Central Asian Orogenic Belt: insights from low-temperature thermochronology. Gondwana Res. 43, 107-122. https://doi.org/10.1016/j.gr.2015.10.004.

Green, P.F., Duddy, I.R., Gleadow, A.J.W., Tingate, P.R., Laslett, G.M., 1986. Thermal annealing of fission tracks in apatite. Chem. Geol. Isot. Geosci. Sect. 59, 237-253. https://doi. org/10.1016/0168-9622(86)90074-6.

Griffis, N.P., Montañez, I.P., Fedorchuk, N., Isbell, J., Mundil, R., Vesely, F., Weinshultz, L. Iannuzzi, R., Gulbranson, E., Taboada, A., Pagani, A., Sanborn, M.E., Huyskens, M., Wimpenny, J., Linol, B., Yin, Q.Z., 2018. Isotopes to ice: constraining provenance of glacial deposits and ice centers in west-central Gondwana. Palaeogeogr. Palaeoclimatol. Palaeoecol., 1-14 https://doi.org/10.1016/j.palaeo.2018.04.020.

Hackspacher, P.C., Ribeiro, L.F.B., Ribeiro, M.C.S., Fetter, A.H., Hadler Neto, J.C., Tello, C.E.S. Dantas, E.L., 2004. Consolidation and break-up of the South American Platform in southeastern Brazil: tectonothermal and denudation histories. Gondwana Res. 7, 91-101. https://doi.org/10.1016/S1342-937X(05)70308-7.

Hallet, B., Hunter, L., Bogen, J., 1996. Rates of erosion and sediment evacuation by glaciers: a review of field data and their implications. Glob. Planet. Change 12, 213-235. https://doi.org/10.1029/2004JF000189.

Harman, R., Gallagher, K., Brown, R., Raza, A., Bizzi, L., 1998. Accelerated denudation and tectonic/geomorphic reactivation of the cratons of northeastern Brazil during the Late Cretaceous. J. Geophys. Res. 103, 27091-27105. https://doi.org/10.1029/ 98JB02524.

Hasui, Y., Almeida, F.F.M., 1970. Geocronologia do centro-oeste brasileiro. Bol. Soc. Bras. Geol. 19, 5-26.

Hasui, Y., Sadowski, G.R., Suguio, K., Fuck, G.F., 1975. The Phanerozoic tectonic Evolution of the Western Minas Gerais State. Academia Brasileira Ciências, Anais, pp. 431-438.

Heilbron, M. Valeriano, C.M. Tassinari, C.C.G. Almeida, J. Tupinambá, M., Siga, O, Trouw, R., 2008. Correlation of Neoproterozoic terranes between the Ribeira Belt, SE Brazil and its African counterpart: comparative tectonic evolution and open questions. Geological Society, London, Special Publications, pp. 211-237 https://oi.org/10.1144/ SP294.12.

Heilbron, M., Cordani, U.G., Alkmim, F.F., Reis, H.L.S., 2017. In: Heilbron, Mônica, Cordani, U.G., Alkmim, F.F. (Eds.), Tectonic genealogy of a miniature continent. Springer, São Francisco Craton, Eastern Brazil, pp. 321-331 https://doi.org/10.1007/978-3-31901715-0.

Heine, C., Zoethout, J., Müller, R.D., 2013. Kinematics of the South Atlantic rift. Solid Earth 4, 215-253. https://doi.org/10.5194/se-4-215-2013.

Heredia, N., García-Sansegundo, J., Gallastegui, G., Farias, P., Giacosa, R., Hongn, F., Tubía, J.M., Alonso, J.J., Busquets, P., Charrier, R., Clariana, P., Colombo, F., Cuesta, A., Gallastegui, J., Giambiagi, L., González-Menéndez, L., Limarino, O., Martín-González, F. Pedreira, D., Quintana, L., Rodríguez-Fernández, L.R., Rubio-Ordóñez, Á., Seggiaro, R., Serra-Varela, S., Spalletti, L., Cardó, R., Ramos, V.A., 2018. The Pre-Andean phases of construction of the Southern Andes basement in Neoproterozoic-Paleozoic Times. In: Folguera, A, Contreras-Reyes, E., Heredia, N., Encinas, A., Iannelli, S.B., Oliveros, V., Arriagada, C. (Eds.), The Evolution of the Chilean-Argentinean Andes. Springer, pp. 111-131 https://doi.org/10.1007/978-3-319-67774-3_5.

Hueck, M., Dunkl, I., Heller, B., Basei, M.A.S., Siegesmund, S., 2018. (U-Th)/He thermochronology and zircon radiation damage in the south american passive margin: thermal overprint of the paraná LIP? Tectonics 37, 4068-4085. https://doi.org/ 10.1029/2018TC005041.

Hueck, M. Dunkl, I, Oriolo, S., Wemmer, K Basei, MA.S, Siegesmund, S, 2019. Comparing contiguous high- and low-elevation continental margins: New (U-Th)/He constraints from South Brazil and an integration of the thermochronological record of the southeastern passive margin of South America. Tectonophysics 770, 228222. https://doi.org/10.1016/j.tecto.2019.228222.

Huismans, R.S., Beaumont, C., 2005. Effect of litospheric stratificationon extensional styles and rift basin geometry. In: Post, P. (Ed.), 25th Annual GCSSEPM Foundation Bob F. Perkins Research Conference Proceedings. Petroleum Systems of Divergent Continental Margin Basins, p. 2005

Hurford, A.J., Green, P.F., 1982. A users' guide to fission track dating calibration. Earth Planet. Sci. Lett. 59, 343-354. https://doi.org/10.1016/0012-821X(82)90136-4.

Hurford, A.J., Green, P.F., 1983. The zeta age calibration of fission-track dating. Isot. Geosci. 1, 285-317. https://doi.org/10.1016/S0009-2541(83)80026-6.

Japsen, P., Bonow, J.M., Green, P.F., Cobbold, P.R., Chiossi, D., Lilletveit, R., Magnavita, L.P. Pedreira, A., 2012. Episodic burial and exhumation in NE Brazil after opening of the South Atlantic. Bull. Geol. Soc. Am. 124, 800-816. https://doi.org/10.1130/B30515.1.

Jelinek, A.R., Chemale, F., van der Beek, P.A., Guadagnin, F., Cupertino, J.A., Viana, A., 2014 Denudation history and landscape evolution of the northern East-Brazilian continental margin from apatite fission-track thermochronology. J. South Am. Earth Sci. 54, 158-181. https://doi.org/10.1016/j.jsames.2014.06.001.

Jonckheere, R., 2003. On methodical problems in estimating geological temperature and time from measurements of fission tracks in apatite. Radiat. Meas. 36, 43-55. https://doi.org/10.1016/S1350-4487(03)00096-9.

Karl, M., Glasmacher, U.A., Kollenz, S., Franco-Magalhaes, A.O.B., Stockli, D.F., Hackspacher P.C., 2013. Evolution of the South Atlantic passive continental margin in southern Brazil derived from zircon and apatite (U-Th-Sm)/He and fission-track data. Tectonophysics 604, 224-244. https://doi.org/10.1016/j.tecto.2013.06.017. 
Kasanzu, C.H., 2017. Apatite fission track and (U-Th)/He thermochronology from the Archean Tanzania Craton: contributions to cooling histories of Tanzanian basement rocks. Geosci. Front. 8, 999-1007. https://doi.org/10.1016/j.gsf.2016.09.007.

Kasanzu, C.H., Linol, B., de Wit, M.J., Brown, R., Persano, C., Stuart, F.M., 2016. From source to sink in central Gondwana: Exhumation of the Precambrian basement rocks of Tanzania and sediment accumulation in the adjacent Congo basin. Tectonics 35, 2034-2051. https://doi.org/10.1002/2016TC004147.

Kohn, B., Gleadow, A., 2019. Application of Low-Temperature Thermochronology to Craton Evolution. In: Malusà, M.G., Fitzgerald, P.G. (Eds.), Fission-track Thermochronology and Its Application to Geology. Springer Textbooks in Earth Sciences, Geography and Environment, pp. 373-393 https://doi.org/10.1007/978-3-319-89421-8_21.

Krabbendam, M., 2001. When the Wilson cycle breaks down: how orogens can produce strong lithosphere and inhibit their future reworking. In: Miller, J.A., Holdsworth, R.E., Buick, I.S., Hand, M. (Eds.), Continental Reactivation and Reworking. Geological Society, pp. 57-75.

Krob, F.C., Glasmacher, U.A., Karl, M., Perner, M., Hackspacher, P.C., Stockli, D.F., 2019. Multi-chronometer thermochronological modelling of the Late Neoproterozoic to recent t-T-evolution of the SE coastal region of Brazil. J. South Am. Earth Sci. 92, 77-94. https://doi.org/10.1016/j.jsames.2019.02.012.

Krob, F.C., Glasmacher, U.A., Bunge, H.-P., Friedrich, A.M., Hackspacher, P.C., 2020. Application of stratigraphic frameworks and thermochronological data on the Mesozoic SW Gondwana intraplate environment to retrieve the Paraná-Etendeka plume movement. Gondwana Res. 84, 81-110. https://doi.org/10.1016/j.gr.2020.02.010.

Kusznir, N.J., Park, R.G., 1984. Intraplate lithosphere deformation and the strength of the lithosphere. Geophys. J. Int. 79, 513-538. https://doi.org/10.1111/j.1365-246X.1984. tb02238.x.

Le Heron, D.P., 2018. An exhumed Paleozoic glacial landscape in Chad. Geology 46, 91-94. https://doi.org/10.1130/G39510.1.

Limarino, C.O., Césari, S.N., Spalletti, L.A., Taboada, A.C., Isbell, J.L., Geuna, S., Gulbranson, E.L., 2014. A paleoclimatic review of southern South America during the late Paleozoic: a record from icehouse to extreme greenhouse conditions. Gondwana Res. 25, 1396-1421. https://doi.org/10.1016/j.gr.2012.12.022

Linol, B., Milani, E.J., François, G., dos Scherer, C.M.S., 2015. New regional correlations between the Congo, Paraná and Cape-Karoo Basins of Southwest Gondwana. In: Oberhansli, R., de Wit, M.J., Roure, F.M. (Eds.), Geology and Resource Potential of the Congo Basin. Springer, Verlag Berlin Heidelberg, pp. 245-268 https://doi.org/ 10.1007/978-3-642-29482-2.

Lobato, G., Borghi, L., 2005. Análise estratigráfica da Formação Furnas (Devoniano Inferior) em afloramentos da borda Leste da Bacia Do Paraná. $3^{\circ}$ Congresso Brasileiro de P\&D Em Petróleo e Gás.

Machado, J.P.S.L., Jelinek, A.R., Bicca, M.M., Stephenson, R., Genezini, F.A., 2019. West Gondwana orogenies and Pangaea break-up: thermotectonic effects on the southernmost Mantiqueira Province, Brazil. J. Geol. Soc. Lond. 176, 1056-1075. https://doi.org/ 10.1144/jgs2019-018.

Mackintosh, V., Kohn, B., Gleadow, A., Gallagher, K., 2019. Long-term reactivation and morphotectonic history of the Zambezi Belt, northern Zimbabwe, revealed by multi-method thermochronometry. Tectonophysics 750, 117-136. https://doi.org/ 10.1016/j.tecto.2018.11.009.

Manatschal, G., Lavier, L., Chenin, P., 2015. The role of inheritance in structuring hyperextended rift systems: some considerations based on observations and numerical modeling. Gondwana Res. 27, 140-164. https://doi.org/10.1016/j.gr.2014.08.006.

Marshak, S., Alkmim, F.F., Whittington, A., Pedrosa-Soares, A.C., 2006. Extensional collapse in the Neoproterozoic Araçuaí orogen, eastern Brazil: a setting for reactivation of asymmetric crenulation cleavage. J. Struct. Geol. 28, 129-147. https://doi.org/ 10.1016/j.jsg.2005.09.006.

Martins-Ferreira, M.A.C., Dias, A.N.C., Chemale, F., Campos, J.E.G., 2020. Intracontinental uplift of the Brazilian Central Plateau linked to continental breakup, orogenies, and basin filling, supported by apatite and zircon fission-track data. Arab. J. Geosci. 13. https://doi.org/10.1007/s12517-020-05885-8.

Martins-Neto, M.A., Pedrosa-Soares, A.C., Lima, S.A.A., 2001. Tectono-sedimentary evolution of sedimentary basins from Late Paleoproterozoic to Late Neoproterozoic in the São Francisco craton and Araçuaí fold belt, eastern Brazil. Sediment. Geol. 141-142, 343-370. https://doi.org/10.1016/S0037-0738(01)00082-3.

Mendes, R.S., 2017. Petrografia, geoquímica, geologia isotópica e geocronologia dos diques máficos do sul do estado do Espírito Santo, Orógeno Araçuaí. Master's thesis. Universidade Federal de Minas Gerais.

Milani, E.J., De Wit, M.J., 2008. Correlations between the classic Paraná and Cape-Karoo sequences of South America and southern Africa and their basin infills flanking the Gondwanides: du Toit revisited. Geol. Soc. Lond. Spec. Publ. 294, 319-342. https:// doi.org/10.1144/SP294.17.

Milani, E.J., Ramos, V.A., 1998. Paleozoic orogenies in southwestern domain of Gondwana and the subsidence cycles of the Paraná Basin. Rev. Bras. Geoci. 28, 473-484. https:// doi.org/10.5327/rbg.v28i4.651.

Milani, E.J., Melo, J.H.G., Souza, P.A., Fernandes, L.A., França, A.B., 2007. Bacia do Paraná. Boletim de Geociências Da Petrobras, pp. 265-287.

Misra, A.A., Mukherjee, S., 2015. Tectonic Inheritance in Continental Rifts and Passive Margins, Springer Briefs in Earth Sciences. Springer International Publishing https:// doi.org/10.1007/978-3-319-20576-2.

Mohriak, W.U., Fainstein, R., 2012. Phanerozoic regional geology of the eastern Brazilian margin. In: Roberts, D.G., Bally, A.W. (Eds.), Regional Geology and Tectonics: Phanerozoic Passive Margins, Cratonic Basins and Global Tectonic Maps. Elsevier, pp. 222-282 https://doi.org/10.1016/B978-0-444-56357-6.00006-8.

Mohriak, W., Nemčok, M., Enciso, G., 2008. South Atlantic divergent margin evolution: rift-border uplift and salt tectonics in the basins of SE Brazil. In: Pankhurst, R.J., Trouw, R.A.J., de Neve, B.B., de Wit, M.J. (Eds.), West Gondwana: Pre-Cenozoic
Correlations Across the South Atlantic Region. Geological Society, London, pp. 365-398 https://doi.org/10.1144/SP294.

Montañez, I.P., Poulsen, C.J., 2013. The Late Paleozoicice age: an evolving paradigm. Annu. Rev. Earth Planet. Sci. 41, 629-656. https://doi.org/10.1146/annurev.earth.031208.100118.

Mooney, W.D., Ritsema, J., Hwang, Y.K., 2012. Crustal seismicity and the earthquake catalog maximum moment magnitude (Mcmax) in stable continental regions (SCRs): correlation with the seismic velocity of the lithosphere. Earth Planet. Sci. Lett. 357-358, 78-83. https://doi.org/10.1016/j.epsl.2012.08.032.

Moraes, R., Brown, M., Fuck, R.A., Camargo, M.A., Lima, T.M., 2002. Characterization and PT Evolution of Melt-bearing Ultrahigh-temperature Granulites: an example from the Anapolis-Itaucu Complex of the Brasilia Fold Belt, Brazil. J. Petrol. 43, 1673-1705. https://doi.org/10.1093/petrology/43.9.1673.

Morais Neto, J.M., Hegarty, K.A., Karner, G.D., Alkmim, F.F., 2009. Timing and mechanisms for the generation and modification of the anomalous topography of the Borborema Province, northeastern Brazil. Mar. Pet. Geol. 26, 1070-1086. https://doi.org/10.1016/ j.marpetgeo.2008.07.002.

Morgan, W.J., 1971. Convection plumes in the Lower Mantle. Nature 230, 42-43.

Morgan, P., 1983. Constraints on Rift thermal processes from heat flow and uplift. Tectonophysics 94, 277-298. https://doi.org/10.1016/B978-0-444-42198-2.50023-7.

Mottin, T.E., Vesely, F.F., de Rodrigues, M.C., Kipper, F., Souza, P.A., 2018. The paths and timing of late Paleozoic ice revisited: new stratigraphic and paleo-ice flow interpretations from a glacial succession in the upper Itararé Group (Paraná Basin, Brazil). Palaeogeogr. Palaeoclimatol. Palaeoecol. 490, 488-504. https://doi.org/10.1016/j. palaeo.2017.11.031

Nachtergaele, S., Glorie, S., Morley, C., Charusiri, P., Kanjanapayont, P., Vermeesch, P., Carter, A., Van Ranst, G., De Grave, J., 2020. Cenozoic tectonic evolution of southeastern Thailand derived from low-temperature thermochronology. J. Geol. Soc. Lond. https://doi.org/10.1144/jgs2018-167 jgs2018-167.

Nemčok, M., 2016. Rifts and Passive Margins: Structural Architecture, Thermal Regimes, and Petroleum Systems. Cambridge University Press.

Oliveira, C.H.E., Jelinek, A.R., Chemale, F., Bernet, M., 2016. Evidence of post-Gondwana breakup in Southern Brazilian Shield: insights from apatite and zircon fission track thermochronology. Tectonophysics 666, 173-187. https://doi.org/10.1016/j. tecto.2015.11.005.

Pankhurst, R.J., Rapela, C.W., Fanning, C.M., Márquez, M., 2006. Gondwanide continental collision and the origin of Patagonia. Earth Sci. Rev. 76, 235-257. https://doi.org/ 10.1016/j.earscirev.2006.02.001.

Peace, A.L., Phethean, J.J.J., Franke, D., Foulger, G.R., Schiffer, C., Welford, J.K., McHone, G. Rocchi, S., Schnabel, M., Doré, A.G., 2019. Publisher's note. Earth Sci. Rev. 196, 102865. https://doi.org/10.1016/j.earscirev.2019.05.009.

Pedrosa-Soares, A.C., Wiedemann-Leonardos, C., 2000. Evolution of the Araçuaí belt and its connection to the Ribeira belt, eastern Brazil. In: Cordani, U.G., Milani, E.J., Thomaz, F., Campos, D.A. (Eds.), Tectonic Evolution of South America, 31st International Geological Congress. Rio de Janeiro, pp. 265-285.

Pedrosa-Soares, A.C., Noce, C.M., Vidal, P., Monteiro, R.L.B.P., Leonardos, O.H. 1992. Toward a new tectonic model for the Late Proterozoic Araçuaí (SE Brazil)-West Congolian (SW Africa) Belt. J. South Am. Earth Sci. 6, 33-47. https://doi.org/ 10.1016/0895-9811(92)90015-Q.

Pedrosa-Soares, A., Noce, C., Wiedemann, C.M., Pinto, C.P., 2001. The Araçaí-West-Congo Orogen in Brazil: an overview of a confined orogen formed during Gondwanaland assembly. Precambrian Res. 110, 307-323. https://doi.org/10.1016/S0301-9268(01) 00174-7.

Pedrosa-Soares, A.C., Alkmim, F.F., Tack, L., Noce, C.M., Babinski, M., Silva, L.C., MartinsNeto, M.A., 2008. In: Pankhurst, R.J., Trouw, R.A.J., Brito Neves, B.B., de Wit, M.J. (Eds.), Similarities and Differences Between the Brazilian and African Counterparts of the Neoproterozoic Araçuaí-West Congo Orogen. Geological Society, London, pp. 153-172 https://doi.org/10.1144/SP294.9.

Pedrosa-Soares, A.C., De Campos, C.P., Noce, C., Silva, L.C., Novo, T., Roncato, J., Medeiros, S., Castañeda, C. Queiroga, G. Dantas, E., Dussin, I., Alkmim, F., 2011. Late Neoproterozoic-Cambrian granitic magmatism in the Araçuaí orogen (Brazil), the Eastern Brazilian Pegmatite Province and related mineral resources. Geol. Soc. Lond. Spec. Publ. 350, 25-51. https://doi.org/10.1144/SP350.3.

Pérez-Díaz, L., Eagles, G., 2014. Constraining South Atlantic growth with seafloor spreading data. Tectonics 33, 1848-1873. https://doi.org/10.1002/2014TC003644.

Peslier, A.H., Woodland, A.B., Bell, D.R., Lazarov, M., 2010. Olivine water contents in the continental lithosphere and the longevity of cratons. Nature 467, 78-81. https://doi. org/10.1038/nature09317.

Petersen, K.D., Schiffer, C., 2016. Wilson cycle passive margins: control of orogenic inheritance on continental breakup. Gondwana Res. 39, 131-144. https://doi.org/10.1016/ j.gr.2016.06.012.

Pimentel, M.M., 2016. The tectonic evolution of the Neoproterozoic Brasília Belt, central Brazil: a geochronological and isotopic approach. Brazil. J. Geol. 46, 67-82. https:// doi.org/10.1590/2317-4889201620150004.

Pimentel, M., Fuck, R., Jost, H., Filho, C., Araújo, S., 2000. The Basement of the Brasília Fold Belt and the Goiás Magmatic Arc. In: Cordani, U.G., Milani, E.J., Thomaz Filho, A., Campos, D.A. (Eds.), Tectonic Evolution of South America. Rio de Janeiro, pp. 195-229 https://doi.org/10.1016/j.ijms.2013.07.006.

Pimentel, M.M., Jost, H., Fuck, R.A., 2004. O embasamento da Faixa Brasília e o Arco Magmático de Goiás. In: Mantesso-Neto, V., Bartorelli, A., Carneiro, C.D.R., Brito Neves, B.B. (Eds.), Geologia Do Continente Sul-Americano: Evolução Da Obra de Fernando Flávio Marques de Almeida. Editora Beca, pp. 324-355.

Pinet, N., 2018. The Ups and Downs of the Canadian Shield: 2- Preliminary Results of Apatite Fission-track Analysis From a 3.6 km Vertical Profile, LaRonde mine, Quebec. https://doi.org/10.4095/308067.

Piuzana, D., 2003. Neoproterozoic granulite facies metamorphism and coeval granitic magmatism in the Brasilia Belt, Central Brazil: regional implications of new SHRIMP 
U-Pb and Sm-Nd data. Precambr. Res. 125, 245-273. https://doi.org/10.1016/S03019268(03)00108-6.

Quirk, D.G., Hertle, M., Jeppesen, J.W., Raven, M., Mohriak, Webster Ueipass, Kann, D.J., Nørgaard, M., Howe, M.J., Hsu, D., Coffey, B., Mendes, M.P., 2013. Rifting, subsidence and continental break-up above a mantle plume in the central South Atlantic. In: Mohriak, W.U., Danforth, A., Post, P.J., Brown, D.E., Tari, G.C., Nemčok, M., Sinha, S.T. (Eds.), Conjugate Divergent Margins. Geological Society, London, pp. 185-214 https://doi.org/10.1144/sp369.20.

Redfield, T.F., 2010. On apatite fission track dating and the Tertiary evolution of West Greenland topography. J. Geol. Soc. Lond. 167, 261-271. https://doi.org/10.1144/ 0016-76492009-036.

Reis, H.L.S., Alkmim, F.F., Fonseca, R.C.S., Nascimento, T.C., Suss, J.F., Prevatti, L.D., 2017. The São Francisco Basin. In: Heilbron, M., Cordani, U.G., Alkmim, Fernando F. (Eds.), São Francisco Craton, Eastern Brazil. Springer, pp. 117-143 https://doi.org/10.1007/9783-319-01715-0.

Reno, B.L., Brown, M., Kobayashi, K., Nakamura, E., Piccoli, P.M., Trouw, R.A.J., 2009. Eclogite-high-pressure granulite metamorphism records early collision in West Gondwana: new data from the Southern Brasília Belt, Brazil. J. Geol. Soc. London. 166, 1013-1032. https://doi.org/10.1144/0016-76492008-140.

Reuber, K., Mann, P., 2019. Control of Precambrian-to-Paleozoic orogenic trends on alongstrike variations in Early Cretaceous continental rifts of the South Atlantic Ocean. Interpretation 7, SH45-SH69. https://doi.org/10.1190/INT-2018-0257.1.

Rey, P., Vanderhaeghe, O., Teyssier, C., 2001. Gravitational collapse of the continental crust: definition, regimes and modes. Tectonophysics 342, 435-449. https://doi.org/ 10.1016/S0040-1951(01)00174-3.

Ribeiro, L.F.B., Hackspacher, P.C., Ribeiro, M.C.S., Hadler Neto, J.C., Tello, S.C.A., Iunes, P.J., Franco, A.O.B., Godoy, D.F., 2005. Thermotectonic and fault dynamic analysis of Precambrian basement and tectonic constraints with the Parana basin. Radiat. Meas. 39, 669-673. https://doi.org/10.1016/j.radmeas.2004.09.007.

Riccomini, C., Velázquez, V.F., Gomes, C.B., 2005. Tectonic controls of the Mesozoic and Cenozoic alkaline magmatism in the central- southeastern Brazilian Platform. In: Comin-Chiaramonti, P. Gomes, C.B. (Eds.), Mesozoic to Cenozoic Alkaline Magmatism in the Brazilian Platform. Editora da Universidade de São Paulo, São Paulo, pp. 31-57.

Richter, F., Lana, C., Stevens, G., Buick, I., Pedrosa-Soares, A.C., Alkmim, F.F., Cutts, K. 2016. Sedimentation, metamorphism and granite generation in a back-arc region: records from the Ediacaran Nova Venécia Complex (Araçuaí Orogen, Southeastern Brazil). Precambr. Res. 272, 78-100. https://doi.org/10.1016/j. precamres.2015.10.012.

Rocha, B.C., Davies, J.H.F.L., Janasi, V.A., Schaltegger, U., Nardy, A.J.R., Greber, N.D., Lucchetti, A.C.F., Polo, L.A., 2020. Rapid eruption of silicic magmas from the Paraná magmatic province (Brazil) did not trigger the Valanginian event. Geology, 1-5 https://doi.org/10.1130/G47766.1.

Rosset, A., De Min, A., Marques, L.S., Macambira, M.J.B., Ernesto, M., Renne, P.R., Piccirillo, E.M., 2007. Genesis and geodynamic significance of Mesoproterozoic and Early Cretaceous tholeiitic dyke swarms from the São Francisco craton (Brazil). J. South Am. Earth Sci. 24, 69-92. https://doi.org/10.1016/j.jsames.2007.02.002.

Rouby, D., Braun, J., Robin, C., Dauteuil, O., Deschamps, F., 2013. Long-term stratigraphic evolution of Atlantic-type passive margins: a numerical approach of interactions between surface processes, flexural isostasy and 3D thermal subsidence. Tectonophysics 604, 83-103. https://doi.org/10.1016/j.tecto.2013.02.003.

Salazar-Mora, C.A., Huismans, R.S., Fossen, H., Egydio-Silva, M., 2018. The Wilson cycle and effects of tectonic structural inheritance on rifted passive margin formation. Tectonics 37, 3085-3101. https://doi.org/10.1029/2018TC004962.

Salomon, E., Koehn, D., Passchier, C., Hackspacher, P.C., Glasmacher, U.A., 2015. Contrasting stress fields on correlating margins of the South Atlantic. Gondwana Res. 28, 1152-1167. https://doi.org/10.1016/j.gr.2014.09.006.

Salomon, E., Passchier, C., Koehn, D., 2017. Asymmetric continental deformation during South Atlantic rifting along southern Brazil and Namibia. Gondwana Res. 51, 170-176. https://doi.org/10.1016/j.gr.2017.08.001.

Santiago, R., de Caxito, F.A., Neves, M.A., Dantas, E.L., Medeiros Júnior, E.B., Queiroga, G.N., 2019. Two generations of mafic dyke swarms in the Southeastern Brazilian coast: reactivation of structural lineaments during the gravitational collapse of the AraçuaíRibeira Orogen (500 Ma) and West Gondwana breakup (140 Ma). Precambrian Res., 105344 https://doi.org/10.1016/j.precamres.2019.105344.

Scherer, C.M.S., Lavina, E.L.C., 2006. Stratigraphic evolution of a fluvial-eolian succession: the example of the Upper Jurassic-Lower Cretaceous Guará and Botucatu formations, Paraná Basin, Southernmost Brazil. Gondwana Res. 9, 475-484. https://doi.org/ 10.1016/j.gr.2005.12.002.

Schiffer, C., Doré, A.G., Foulger, G.R., Franke, D., Geoffroy, L., Gernigon, L., Holdsworth, B., Kusznir, N., Lundin, E., McCaffrey, K., Peace, A.L., Petersen, K.D., Phillips, T.B., Stephenson, R., Stoker, M.S., Welford, J.K., 2019. Structural inheritance in the North Atlantic. Earth Sci. Rev., 102975 https://doi.org/10.1016/j.earscirev. 2019.102975.

Sgarbi, G.N.C., Sgarbi, P.B.A., Campos, J.E.G., Dardenne, M.A., Penha, U.C., 2001. Bacia Sanfranciscana: O registro Fanerozóico da Bacia do São Francisco. In: Pinto, C.P., Martins-Neto, M.A. (Eds.), Bacia Do São Francisco: Geologia e Recursos Naturais. Soc. Bras. Geol. (SBG) - Núcleo MG, Belo Horizonte.

Sleep, N.H., 2003. Survival of Archean cratonal lithosphere. J. Geophys. Res. Solid Earth 108, 1-29. https://doi.org/10.1029/2001jb000169.

Soares, J.E., Berrocal, J., Fuck, R.A., Mooney, W.D., Ventura, D.B.R., 2006. Seismic characteristics of central Brazil crust and upper mantle: a deep seismic refraction study. J. Geophys. Res. Solid Earth 111, 1-31. https://doi.org/10.1029/2005JB003769.

Souza, D.H., Hackspacher, P.C., Doranti-Tiritan, C., Godoy, D.F., 2014. Comparison of long and short term evolutionary dynamics, between the Poços de Caldas plateau and the São Pedro de Caldas plateau. Rev. Bras. Geomorfol. 16, 251-272.
Spier, C.A.,Vasconcelos, P.M.,Oliviera, S.M.B., 2006.40Ar/39Argeochronological constraints on the evolution of lateritic iron deposits in the Quadrilátero Ferrífero, Minas Gerais, Brazil. Chem. Geol. 234, 79-104. https://doi.org/10.1016/j.chemgeo.2006.04.006.

Stanley, J.R., Flowers, R.M., Bell, D.R., 2015. Erosion patterns and mantle sources of topographic change across the southern African Plateau derived from the shallow and deep records of kimberlites. Geochem. Geophys. Geosyst. 16, 3235-3256. https:// doi.org/10.1002/2015GC005969.

Thompson, R.N., Gibson, S.A., Mitchell, J.G., Dickin, A.P., Leonardos, O.H., Brod, J.A., Greenwood, J.C., 1998. Migrating Cretaceous-eocene magmatismin the Serra Do Mar Alkaline Province, Se Brazil: melts from the deflected trindade mantle plume? J. Petrol. 39, 1493-1526. https://doi.org/10.1093/petroj/39.8.1493.

Tohver, E., D'Agrella-Filho, M.S., Trindade, R.I.F., 2006. Paleomagnetic record of Africa and South America for the 1200-500 Ma interval, and evaluation of Rodinia and Gondwana assemblies. Precambrian Res. 147, 193-222. https://doi.org/10.1016/j. precamres.2006.01.015

Tommasi, A., Vauchez, A., 1997. Continental-scale rheological heterogeneities and complex intraplate tectono-metamorphic patterns: insights from a case-study and numerical models. Tectonophysics 279, 327-350. https://doi.org/10.1016/S0040-1951 (97)00117-0.

Tommasi, A., Vauchez, A., 2001. Continental rifting parallel to ancient collisional belts: an effect of the mechanical anisotropy of the lithospheric mantle. Earth Planet. Sci. Lett. 185, 199-210. https://doi.org/10.1016/S0012-821X(00)00350-2.

Tommasi, A., Vauchez, A., 2015. Heterogeneity and anisotropy in the lithospheric mantle. Tectonophysics 661, 11-37. https://doi.org/10.1016/j.tecto.2015.07.026.

Torsvik, T.H., Cocks, L.R.M., 2013. Gondwana from top to base in space and time. Gondwana Res. 24, 999-1030. https://doi.org/10.1016/j.gr.2013.06.012.

Torsvik, T.H., Rousse, S., Labails, C. Smethurst, M.A., 2009. A new scheme for the opening of the South Atlantic Ocean and the dissection of an Aptian salt basin. Geophys. J. Int. 177, 1315-1333. https://doi.org/10.1111/j.1365-246X.2009.04137.x.

Trompette, R., 1994. Geology of Western Gondwana (2000-500 Ma): Pan-AfricanBrasiliano Aggregation of South America and Africa. Rotterdam.

Trompette, R.R., Uhlein, A., Silva, M.E., Karmann, I., 1992. O Cráton Brasiliano Do São Francisco - Uma Revisão. Rev. Bras. Geoci. 22, 481-486. https://doi.org/10.25249/ 0375-7536.1991481486.

Turner, J.P., Green, P.F., Holford, S.P., Lawrence, S.R., 2008. Thermal history of the Rio Mun (West Africa)-NE Brazil margins during continental breakup. Earth Planet. Sci. Lett. 270, 354-367. https://doi.org/10.1016/j.epsl.2008.04.002.

Uhlein, A., de Freitas, A.M., da Cruz, A.B., da Silva, W.F., de Caxito, F.A., de Moreira, G.C., 2011. Projeto Alto Paranaíba - Folha Carmo do Paranaíba (Belo Horizonte).

Van Ranst, G., Pedrosa-Soares, A.C., Novo, T., Vermeesch, P., De Grave, J., 2020a. New insights from low-temperature thermochronology into the tectonic and geomorphologic evolution of the south-eastern Brazilian highlands and passive margin. Geosci. Front. 11, 303-324. https://doi.org/10.1016/j.gsf.2019.05.011.

Valeriano, C.M., 2017. The Southern Brasília Belt. In: Heilbron, M., Cordani, U.G., Alkmim, F.F. (Eds.), São Francisco Craton. Springer, Eastern Brazil, pp. 189-203.

Valeriano, C.M., 2017a. The Southern Brasília Belt. São Francisco Craton, Eastern Brazil. Springer, pp. 189-203 https://doi.org/10.1007/978-3-319-01715-0_10.

Valeriano, C.M., Dardenne, M.A., Fonseca, M.A., Simões, L.S.A., Seer, H.J., 2004. A evolução tectônica da Faixa Brasília. In: Mantesso-Neto, V., Bartorelli, A., Carneiro, C.D.R., Brito Neves, B.B. (Eds.), Geologia Do Continente Sul-Americano: Evolução Da Obra de Fernando Flávio de Almeida. Editora Beca, São Paulo, pp. 575-592.

Valeriano, C.M., Teixeira, W., Heilbron, M., Sergio, L., Simões, A., 2000. Southern Brasília Belt (SE Brazil): tectonic discontinuities, $\mathrm{K}-\mathrm{Ar}$ data and evolution during the neoproterozoic Brasiliano orogeny. Rev. Bras. Geoci. 30, 195-199.

Van Ranst, G., Baert, P., Fernandes, A.C., De Grave, J., 2020b. Technical note: TRACKFlow, a new versatile microscope system for fission track analysis. Geochronology 2, 93-99. https://doi.org/10.5194/gchron-2-93-2020.

Vauchez, A., Barruol, G., Tommasi, A., 1997. Why do continents break-up parallel to ancient orogenic belts? Terra Nov. 9, 62-66. https://doi.org/10.1111/j.13653121.1997.tb00003.x

Vauchez, A., Egydio-Silva, M., Babinski, M., Tommasi, A., Uhlein, A., Liu, D., 2007. Deformation of a pervasively molten middle crust: Insights from the neoproterozoic RibeiraAraçuaí orogen (SE Brazil). Terra Nov. 19, 278-286. https://doi.org/10.1111/j.13653121.2007.00747.x

Vauchez, A., Hollanda, M.H.B.M., Monié, P., Mondou, M., Egydio-Silva, M., 2019. Slow cooling and crystallization of the roots of the Neoproterozoic Araçuai hot orogen (SE Brazil): implications for rheology, strain distribution, and deformation analysis. Tectonophysics 766, 500-518. https://doi.org/10.1016/j. tecto.2019.05.013.

Vaughan, A.P.M., Pankhurst, R.J., 2008. Tectonic overview of the West Gondwana margin. Gondwana Res. 13, 150-162. https://doi.org/10.1016/j.gr.2007.07.004.

Vermeesch, P., 2018. IsoplotR: a free and open toolbox for geochronology. Geosci. Front. 9 1479-1493. https://doi.org/10.1016/j.gsf.2018.04.001.

Vesely, F.F., Assine, M.L., 2006. Deglaciation sequences in the Permo-Carboniferous Itarare Group, Paraná Basin, southern Brazil. J. South Am. Earth Sci. 22, 156-168. https://doi. org/10.1016/j.jsames.2006.09.006.

Visser, J.N.J., 1987. The palaeogeography of part of southwestern Gondwana during the Permo-Carboniferous glaciation. Palaeogeogr. Palaeoclimatol. Palaeoecol. 61, 205-219. https://doi.org/10.1016/0031-0182(87)90050-2.

Watts, A.B., 2012. Models for the evolution of passive margins. Reg. Geol. Tectonics Phaneroz. Rift Syst. Sediment. Basins, 32-57 https://doi.org/10.1016/B978-0-44456356-9.00002-X

Weissel, J.K., Karner, G.D., 1989. Flexural uplift of rift flanks due to mechanical unloading of the lithosphere during extension. J. Geophys. Res. Solid Earth 94, 13919-13950. https://doi.org/10.1029/JB094iB10p13919. 
Will, T.M.,Frimmel,H.E., 2018. Where does a continent prefer to breakup? Somelessons from the South Atlantic margins. Gondwana Res. 53, 9-19. https://doi.org/10.1016/j.gr.2017.04.014.

Zalán, P.V., 2004. Evolução fanerozóica das bacias sedimentares Brasileiras. In: MantessoNeto, V., Bartorelli, A. Carneiro, C.D.R., Brito Neves, B.B. (Eds.), Geologia Do Continente Sul-Americano: Evolução Da Obra de Fernando Flávio Marques de Almeida. Beca, São Paulo, pp. 595-613.
Zalán, P.V., Wolff, S., Astolfi, M.A.M., Vieira, I.S., Concelção, J.C.J., Appi, V.T., Neto E.V.S., Cerqueira, J.R., Marques, A., 1990. The Paraná Basin, Brazil. In: Leighton, M.W., Kolata, D.R., Oltz, D.F., Eidel, J.J. (Eds.), Interior Cratonic Basins. Memoir of the American Association of Petroleum Geologists, pp. 681-708. 